Original Article

\title{
DESIGN, SYNTHESIS AND ANTICONVULSANT PROFILE OF 5-(BENZO [D][1,3]DIOXOL-5-YL)-3- TERT-BUTYL-4, 5-DIHYDROPYRAZOLE DERIVATIVES
}

\author{
MOHAMMED FARRAG EL-BEHAIRYa* ${ }^{*}$ HANAN NAEIM HAFEZ ATTIA ${ }^{b}$
}

aMedicinal and Pharmaceutical Chemistry Department, Pharmaceutical and Drug Industries Research Division, National Research Centre (ID 60014618), 12622 Dokki, Giza, Egypt, bMedicinal and Pharmaceutical Chemistry Department (Pharmacology Group), Pharmaceutical and Drug Industries Research Division, National Research Centre (ID 60014618), 12622 Dokki, Giza, Egypt

Email: mohabeha@gmail.com

Received: 11 Jul 2016 Revised and Accepted: 09 May 2017

\section{ABSTRACT}

Objective: Utilisation of the ligand-based design and molecular hybridization to design promising candidates with prospective efficacy and safety. Synthesis of the designed candidates using different synthetic methods. Biological evaluation of the newly synthesised candidates as anticonvulsant agents.

Methods: Three novel series of 5-(benzo[d][1,3]dioxol-5-yl)-3-tert-butyl-4,5-dihydropyrazoles have been designed via ligand-based drug discovery and molecular hybridization. Proper synthetic routes have been followed in the preparation of compounds (2-23) which have been characterised by different spectral techniques. Antiepileptic potential was assessed by biological evaluation using 'classical' animal models of epilepsy, in addition to rotarod test for toxicity.

Results: 4-Nitrophenyl derivatives (5, 13, and19) displayed the highest potency. Compound 5was the most active substituent in series A (N'-aroyl3-tert-butyl-4,5-dihydro-1H-pyrazole-1-carbohydrazide). It was 2.7 and 1.3 times more active than reference drug Stiripentol (I) and lead compound III, respectively. Compound 13 was the best candidate in series B (N'-arylidene-3-tert-butyl-4,5-dihydro-1H-pyrazole-1-carbohydrazide). It was 3.3, 1.5, and 1.2 times more potent than Stiripentol, lead compound III and new compound 5, respectively. Two members (19 and 21) of series C (1,3,4-oxadiazole derivatives) achieved $100 \%$ protection at lower doses than I and III, being 2.6 and 2.4 times more active than Stiripentol. In $s c$ PTZ screen, the most active congeners $(5,13,19)$ exhibited $\mathrm{ED}_{50}$ values of 45,48 , and $81 \mathrm{mg} / \mathrm{kg}$, respectively, which are highly superior as compared to that of reference drug Stiripentol(I) and lead compound III (ED50 115 and $110 \mathrm{mg} / \mathrm{kg}$, respectively).

Conclusion: Ligand-based design together with molecular hybridization in drug design succeeded to produce potent and wide spectrum candidates. Keywords: Stiripentol, Anticonvulsant, Ligand-based drug design, Molecular hybridization, 2-pyrazoline

(C) 2017 The Authors. Published by Innovare Academic Sciences Pvt Ltd. This is an open access article under the CC BY license (http://creativecommons.org/licenses/by/4.0/) DOI: http://dx.doi.org/10.22159/ijpps.2017v9i6.17520

\section{INTRODUCTION}

Epilepsy is the most common neurological disorder after stroke, debilitating around 50 million people worldwide. It is worth noting that $80 \%$ of epileptic patients were diagnosed in developing countries, such as Egypt [1, 2]. In the past two decades, many antiepileptic drugs have been launched, such as carbazepine, levetiracetam, stiripentol, fluoro felbamate, pregabalin, lamotrigine, gabapentin, and topiramate. Unfortunately, these treatments are not prescribed to three out of four patients in developing countries [1]. Moreover, therapy-resistant epilepsy still affects about 1.8 million people worldwide [3]. Convulsions in $30 \%$ of epileptics remain inadequately controlled by available drug therapy [4]. Furthermore, CNS related adverse side effects such as executive function, diminished attention, language skills, memory and processing speed are frequently reported [5]. Accordingly, new generation antiepileptic drugs (AEDs) fail to exhibit ample advantage over the formerly recognised agents. Hence, further search for safer and more effective AEDs is mandatory.
Rational drug design procedures are frequently used for drug discovery of biological targets (with well-known three-dimensional structure). But for new AEDs, ligand-based drug design is the dominant technique, due to the lack of human epilepsy pathogenic data [6]. This technique is based on utilizing present structureactivity relationship (SAR) data of old and new generation AEDs, besides other potent anticonvulsant candidates, followed by structural modifications.

Following the ligand-based design, our group has developed a series of derivatives from stiripentol (I) (an orphan antiepileptic drug with unique activity against severe myoclonic epilepsy in infants (SMEI)/or Dravet's syndrome [7]). Compounds II and III were the most active congeners showing potent activity in MES and $s c$ PTZ screens, respectively. At $350 \mu \mathrm{mol}$, compound III was 2.13 times more active than stiripentol in scPTZ test, while compound II displayed $100 \%$ protection against MES screen at the same dose level, which was unachievable by lead compound (stiripentol I), even at $747 \mu \mathrm{mol}[8]$.

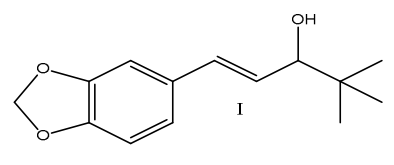

747 umol, $100 \%$ scPTZ

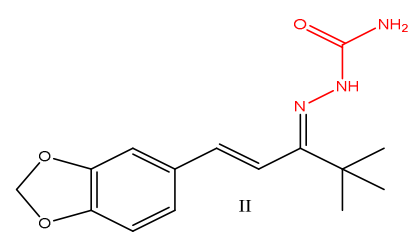

346 umol, $100 \%$ MES

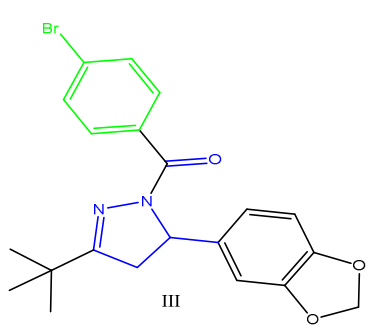

$350 \mathrm{umol}, 100 \% \mathrm{scPTZ}$

Fig. 1: Structures of stiripentol (I) and its active anticonvulsant derivatives II and III [8] 
Compounds II and III represent very good progress in stiripentol activity. However, the spectrum of such compounds was narrow, since compound II which showed $100 \%$ protection in MES test, displayed $50 \%$ protection in $s c$ PTZ screen. Meanwhile, compound III exhibited only $16 \%$ protection in MES but achieved $100 \%$ in $s c$ PTZ test. These results justify the need for further structural optimisations in order to broaden their spectrum of activity. The systematic SAR study of compounds II and III concluded that the presence of the semicarbazone moiety (red color) as non-substituted open chain form (compound II) was very beneficial for the MES activity while its cyclization to aroyl substituted 2-pyrazoline (blue and green color) was enough to favor the activity against scPTZinduced seizures (compound III). Thus, it was very compelling to design a molecule which contains both semicarbazone moiety (red color) and the 2-pyrazoline structure (blue color). This design leads to series A, 5-(benzo [d] [1, 3] dioxol-5-yl)-N'-aroyl-3-tert-butyl-4, 5dihydro-1H-pyrazole-1-carbohydrazide, derivatives (3-10) (fig. 2).
Converting the amide group in series A to imine moiety in series B, 5(benzo[d][1,3]dioxol-5-yl)-N'-arylidene-3-tert-butyl-4,5-dihydro-1Hpyrazole-1-carbohydrazide derivatives (11-16), is satisfactory as well, since it produces typical semicarbazone structure and permits studying the effect of H-bond donors/acceptors on the activity.

Additionally, the influence of cyclizing the aroyl carbohydrazide moiety of series A to 1,3,4-oxadiazole, which is a native anticonvulsant scaffold[9-11]in series C, 2-(5-(benzo[d][1,3]dioxol-5-yl)-3-tert-butyl4,5-dihydro-1H-pyrazol-1-yl)-5-(4-bromo phenyl)-1,3,4-oxadiazole derivatives (17-22), is very intriguing, since it will completely omit the H-bond donors and consequently elaborate its impact on anticonvulsant activity. These designs have followed the pharmacophoric model that has been put forward for antiepileptic activity owing to conformational investigations on prevailing anticonvulsant drugs such as phenytoin, carbamazepine, lamotrigine and phenobarbitone $[12,13]$.
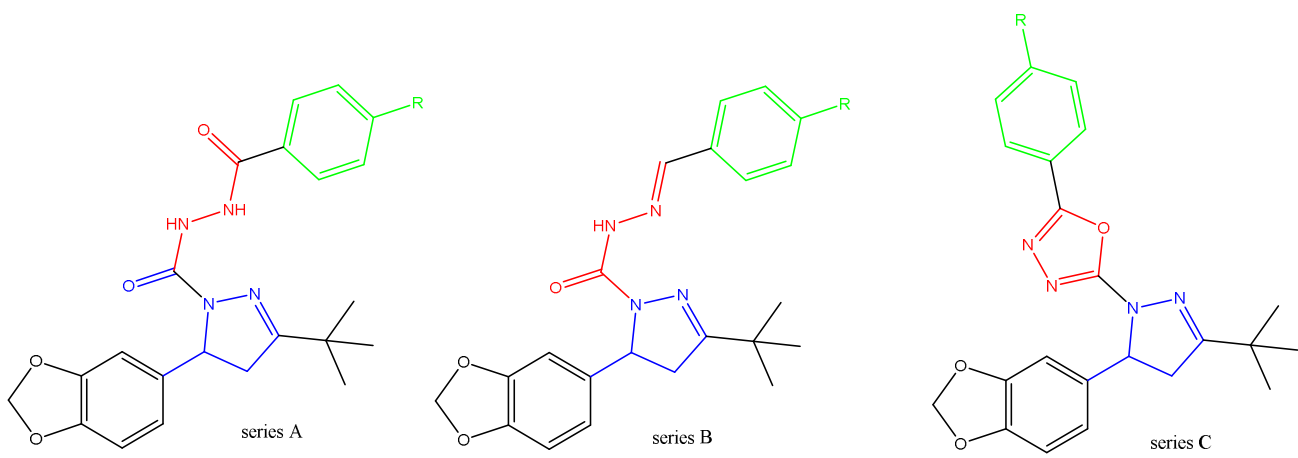

Fig. 2: Structures of the anticipated series A, B, and C showing different pharmacophoric moieties

In view of the aforementioned findings, the aim of the present study was to adopt the ligand-based design and molecular hybridization process in order to obtain promising candidates, with prospective efficacy and safety profile, as compared to reference AEDs (Stiripentol (I), lead compounds II and III). Biological assessment of antiepileptic potential was performed using 'classical' animal models of epilepsy (MES and scPTZ seizure tests), in addition to the neurotoxicity test.

\section{MATERIALS AND METHODS}

\section{Materials}

\section{Drugs and chemicals}

\section{All chemicals utilized in the chemical preparation were of} analytical grade

\section{Chemistry}

All melting points were determined using electrothermal capillary melting point apparatus and are uncorrected. Infrared (IR) spectra were recorded as a thin film (for oils) in $\mathrm{NaCl}$ discs or as $\mathrm{KBr}$ pellets (for solids) with JASCO FT/IR-6100 spectrometer and values are represented in $\mathrm{cm}^{-1} .{ }^{1} \mathrm{H}$ NMR $(300$ and $500 \mathrm{MHz})$ and ${ }^{13} \mathrm{C}$ NMR $(75$ and $125 \mathrm{MHz}$ ) spectra were carried out on Jeol ECA $500 \mathrm{MHz}$ spectrometer and Avance II NMR $300 \mathrm{MHz}$ spectrometers (Bruker Biospin) using TMS as internal standard and chemical shift values were recorded in ppm on $\delta$ scale.

The ${ }^{1} \mathrm{H}$ NMR data were represented as follows: chemical shifts, multiplicity (s. singlet, d. doublet, dd. doublet of the doublet, $t$. triplet, m. multiplet, br. Broad, AR. aromatic), a number of protons. The ${ }^{13} \mathrm{C}$ NMR data were represented as chemical shifts. Mass spectral data were obtained with electron impact (EI) ionisation technique at $70 \mathrm{eV}$ from a Finnigan Mat SSQ-7000 Spectrophotometer. Silica gel TLC (thin layer chromatography) cards from Merck (silica gel precoated aluminium cards with a fluorescent indicator at $245 \mathrm{~nm}$ ) were used for thin layer chromatography. Visualization was performed by illumination with UV light source $(254 \mathrm{~nm})$. Column chromate-graphy was carried out on silica gel $60(0.063-0.200 \mathrm{~mm})$ obtained from Merck.

\section{5-(benzo[d][1,3]dioxol-5-yl)-3-tert-butyl-4,5-dihydro-1H- pyrazole (1)}

Compound 1 has been synthesised according to the reported procedures by Aboul-Enein et al. [8].

Synthesis of 5-(benzo[d][1,3]dioxol-5-yl)-3-tert-butyl-4,5dihydro-1H-pyrazole-1-carbohydrazide (2)

To a stirred solution of $1(2.0 \mathrm{~g}, 8.1 \mathrm{mmol})$ in $20 \mathrm{ml} \mathrm{CHCl}$, phosgene $(12.5 \% \mathrm{w} / \mathrm{v}$ solution in toluene, $0.8 \mathrm{~g}, 6.4 \mathrm{ml}, 8.1 \mathrm{mmol})$ was added. The mixture was stirred at room temperature for $10 \mathrm{~min}$ followed by the addition of hydrazine hydrate $(2.0 \mathrm{~g}$., $2.0 \mathrm{ml}, 40.5 \mathrm{mmol}, 5 \mathrm{~mol}$ equivalents). The reaction mixture was stirred at room temperature for $30 \mathrm{~min}$ then washed with $\mathrm{NaHCO}_{3}(10 \%, 20 \mathrm{ml})$. Water and organic layer were separated, dried $\left(\mathrm{Na}_{2} \mathrm{SO}_{4}\right)$ and evaporated under vacuum to afford $2.4 \mathrm{~g}$ of 2 as white powder (mp 164-166 ${ }^{\mathrm{C}} \mathrm{C}$ ). IR $\left(\mathrm{KBr}, \mathrm{cm}^{-1}\right): 3451\left(\mathrm{NH}_{2}\right), 3354(\mathrm{NH}), 1736(\mathrm{C}=0), 1690(\mathrm{C}=\mathrm{N}) \cdot{ }^{1} \mathrm{H}$ NMR $\left(\mathrm{CDCl}_{3}, 500 \mathrm{MHz}\right): \delta=7.01(\mathrm{~s}, 1 \mathrm{H}, \mathrm{NHCO}), 6.79-6.66(\mathrm{~m}, 3 \mathrm{H}, \mathrm{AR}-$ $\mathrm{H}), 5.88\left(\mathrm{~s}, 2 \mathrm{H}, \mathrm{OCH}_{2} \mathrm{O}\right), 5.30(\mathrm{dd}, J=11.45,5.35 \mathrm{~Hz}, 1 \mathrm{H}$, pyrazoline$\mathrm{H}), 3.66\left(\mathrm{~s}, 2 \mathrm{H}, \mathrm{NH}_{2}\right.$ ), 3.30 (dd, $J=17.6,12.2 \mathrm{~Hz}, 1 \mathrm{H}$, Pyrazoline- $\mathrm{H}$ ), 2.68 (dd, $J=17.6,5.35 \mathrm{~Hz}, 1 \mathrm{H}$, pyrazoline-H), $1.21(\mathrm{~s}, 9 \mathrm{H}$, $\left.\mathrm{C}\left(\mathrm{CH}_{3}\right)_{3}\right) \cdot{ }^{13} \mathrm{C}$ NMR $\left(\mathrm{CDCl}_{3}, 125 \mathrm{MHz}\right) \delta 163.8(\mathrm{C}=\mathrm{N}), 156.9(\mathrm{C}=0), 148.1$ (AR-C), 146.9 (AR-C), 136.9 (AR-C), 118.9 (AR-C), 108.5 (AR-C), 105.8 (AR-C), $101.1 \quad\left(\mathrm{OCH}_{2} \mathrm{O}\right), \quad 60.4 \quad$ (pyrazoline- $\left.\mathrm{CH}\right), \quad 41.7$ (pyrazoline- $\mathrm{CH}_{2}$ ), 34.0 (t-Butyl-C), $28.0\left(\mathrm{CH}_{3}\right)$.

General procedure for synthesis of 5-(benzo[d][1,3]dioxol-5yl)-3-tert-butyl-N'-aroyl-4,5-dihydro-1 $H$-pyrazole-1carbohydrazide (3-10)

To a stirred solution of 2 (2.0 g, $6.5 \mathrm{mmol})$ in chloroform, appropriate acid chloride $(6.5 \mathrm{mmol})$ was added followed by triethylamine ( 0.66 g., $0.92 \mathrm{ml}, 6.5 \mathrm{mmol})$. The reaction mixture was stirred at room temperature for $18 \mathrm{~h}$, washed with $\mathrm{NaHCO}_{3}(10 \%, 20$ $\mathrm{ml})$. Water and organic layer were separated, dried $\left(\mathrm{Na}_{2} \mathrm{SO}_{4}\right)$ and 
evaporated under vacuum to afford crude products, which were further purified by crystallization from ethanol.

\section{5-(benzo[d][1,3]dioxol-5-yl)-3-tert-butyl-N'-benzoyl-4,5- dihydro-1H-pyrazole-1-carbohydrazide (3)}

White solid, yield 63\%, mp $176^{\square} \mathrm{C}$, IR (KBr, cm-1): $3419(\mathrm{NH}), 3231$ (NH), 1641(broad, C=0, C=N). ${ }^{1} \mathrm{H}$ NMR $\left(\mathrm{CDCl}_{3}, 300 \mathrm{MHz}\right) \delta 8.41(\mathrm{~s}$, $1 \mathrm{H}, \mathrm{NHCO}), 8.01$ (s, 1H, NHCO), 7.81 (d, J = 7.07 Hz, 2H, AR-H), 7.57$7.34(\mathrm{~m}, 3 \mathrm{H}, \mathrm{AR}-\mathrm{H}), 6.79-6.66(\mathrm{~m}, 3 \mathrm{H}, \mathrm{AR}-\mathrm{H}), 5.94\left(\mathrm{~s}, 2 \mathrm{H}, \mathrm{OCH}_{2} \mathrm{O}\right)$ $5.30(\mathrm{dd}, J=11.7,5.1 \mathrm{~Hz}, 1 \mathrm{H}$, pyrazoline-H), 3.40 (dd, $J=17.9,11.7$ $\mathrm{Hz}, 1 \mathrm{H}$, pyrazoline-H), 2.80 (dd, $J=17.9,5.1 \mathrm{~Hz}, 1 \mathrm{H}$, pyrazoline-H), $1.26\left(\mathrm{~s}, 9 \mathrm{H}, \mathrm{C}\left(\mathrm{CH}_{3}\right)_{3}\right) \cdot{ }^{13} \mathrm{C} \mathrm{NMR}\left(\mathrm{CDCl}_{3}, 75 \mathrm{MHz}\right) \delta 168.1(\mathrm{C}=\mathrm{N}), 166.3$ (C=0), 156.7 ( $\mathrm{C}=0$ ),148.0 (AR-C), 147.1(AR-C), 138.9 (AR-C), 133.8 (AR-C), 132.9 (AR-C), 129.2 (AR-C), 128.8(AR-C), 119.1 (AR-C), 109.4 (AR-C), 106.5 (AR-C), $100.4\left(\mathrm{OCH}_{2} \mathrm{O}\right), 60.8$ (pyrazoline- $\mathrm{CH}$ ), 41.1 (pyrazoline- $\left.\mathrm{CH}_{2}\right), 35.1$ (t-butyl-C), $27.9\left(\mathrm{CH}_{3}\right) . \quad$ MS: for $\mathrm{C}_{22} \mathrm{H}_{24} \mathrm{~N}_{4} \mathrm{O}_{4}$, calculated. $408.18\left(\mathrm{M}^{+}\right)$, found 408.21 .

\section{5-(benzo[d][1,3]dioxol-5-yl)-3-tert-butyl-N'-(4-bromobenzoyl)-} 4,5-dihydro-1 $H$-pyrazole-1-carbohydrazide $(4)$

White solid, yield 58\%, mp184 ${ }^{\square} \mathrm{C}$, IR $\left(\mathrm{KBr}, \mathrm{cm}^{-1}\right): 3284(\mathrm{NH})$, 1651(broad, $\mathrm{C}=0, \mathrm{C}=\mathrm{N}) \cdot{ }^{1} \mathrm{H}$ NMR $\left(\mathrm{CDCl}_{3}, 300 \mathrm{MHz}\right) \delta 8.13(\mathrm{~d}, J=4.4$ $\mathrm{Hz}, 1 \mathrm{H}, \mathrm{NHCO}), 8.0$ (d, J = 4.41 Hz, $1 \mathrm{H}, \mathrm{NHCO}), 7.68(\mathrm{~d}, J=8.6 \mathrm{~Hz}, 2 \mathrm{H}$, AR-H), 7.58 (d, $J=8.54 \mathrm{~Hz}, 2 \mathrm{H}, \mathrm{AR}-\mathrm{H}), 6.82-6.67(\mathrm{~m}, 3 \mathrm{H}, \mathrm{AR}-\mathrm{H}), 5.96$ (s, $2 \mathrm{H}, \mathrm{OCH}_{2} \mathrm{O}$ ), 5.31 (dd, $J=11.7,5.1 \mathrm{~Hz}, 1 \mathrm{H}$, pyrazoline-H), 3.42 (dd, $J=17.9,11.7 \mathrm{~Hz}, 1 \mathrm{H}$, pyrazoline-H), 2.81 (dd, $J=17.9,5.1 \mathrm{~Hz}, 1 \mathrm{H}$, pyrazoline-H), $1.26\left(\mathrm{~s}, 9 \mathrm{H}, \mathrm{C}\left(\mathrm{CH}_{3}\right)_{3}\right) \cdot{ }^{13} \mathrm{C}$ NMR $\left(\mathrm{CDCl}_{3}, 75 \mathrm{MHz}\right) \delta 165.6$ $(\mathrm{C}=\mathrm{N}), 165.1(\mathrm{C}=0), 153.5(\mathrm{C}=0), 148.0(\mathrm{AR}-\mathrm{C}), 147.0(\mathrm{AR}-\mathrm{C}), 136.1$ (AR-C), 131.5 (AR-C), 130.5 (AR-C), 128.9 (AR-C), 126.6 (AR-C), 118.1 (AR-C), 108.4 (AR-C), 105.8 (AR-C), $101.1\left(\mathrm{OCH}_{2} \mathrm{O}\right), 60.2$ (pyrazoline-CH), 41.9 (pyrazoline- $\left.\mathrm{CH}_{2}\right), 34.1$ (t-butyl-C), $28.0\left(\mathrm{CH}_{3}\right)$. MS: for $\mathrm{C}_{22} \mathrm{H}_{23} \mathrm{BrN}_{4} \mathrm{O}_{4}$, calculated486.09 $\left(\mathrm{M}^{+}\right)$, found 486.22 and $\left(\mathrm{M}^{+}+2\right) 488.20$

\section{5-(benzo[d][1,3]dioxol-5-yl)-3-tert-butyl-N'-(4-nitrobenzoyl)- 4,5-dihydro-1H-pyrazole-1-carbohydrazide (5)}

Yellow solid, yield $84 \%, 228{ }^{\text {घC }}$, IR $\left(\mathrm{KBr}, \mathrm{cm}^{-1}\right)$ : $3386(\mathrm{NH}), 3192$ $(\mathrm{NH}), 1645$ (broad, $\mathrm{C}=0, \mathrm{C}=\mathrm{N}) .{ }^{1} \mathrm{H}$ NMR $\left(\mathrm{CDCl}_{3}, 300 \mathrm{MHz}\right) \delta 8.75(\mathrm{~s}$, $1 \mathrm{H}, \mathrm{NHCO}), 8.28(\mathrm{~d}, J=8.5 \mathrm{~Hz}, 2 \mathrm{H}, \mathrm{AR}-\mathrm{H}), 8.19(\mathrm{~d}, J=8.5 \mathrm{~Hz}, 2 \mathrm{H}, \mathrm{AR}-$ H), $6.71(\mathrm{~d}, J=7.9 \mathrm{~Hz}, 1 \mathrm{H}, \mathrm{AR}-\mathrm{H}), 6.60(\mathrm{dd}, J=7.9,1.8 \mathrm{~Hz}, 1 \mathrm{H}, \mathrm{AR}-\mathrm{H})$, $6.04(\mathrm{~s}, 1 \mathrm{H}, \mathrm{AR}-\mathrm{H}), 5.99$ (dd, $\left.J=13.77,1.4 \mathrm{~Hz}, 2 \mathrm{H}, \mathrm{OCH}_{2} \mathrm{O}\right), 5.32$ (dd, $J$ $=11.3,4.7 \mathrm{~Hz}, 1 \mathrm{H}$, yrazoline- $\mathrm{H}), 3.36(\mathrm{dd}, J=18.3,11.4 \mathrm{~Hz}, 1 \mathrm{H}$, pyrazoline-H), 2.75 (dd, $J=18.4,4.7 \mathrm{~Hz}, 1 \mathrm{H}$, pyrazoline-H), 1.20 (s, $\left.9 \mathrm{H}, \mathrm{C}\left(\mathrm{CH}_{3}\right)_{3}\right) .{ }^{13} \mathrm{C} \mathrm{NMR}\left(\mathrm{CDCl}_{3}, 75 \mathrm{MHz}\right) \delta 165.93(\mathrm{C}=\mathrm{N}), 164.39$ $(\mathrm{C}=0), 154.79$ (C=0), 148.04 (AR-C), 147.0 (AR-C), 136.98 (AR-C), 135.89 (AR-C), 129.21 (AR-C), 128.81 (AR-C), 123.34 (AR-C), 118.76 (AR-C), 108.41 (AR-C), 105.51 (AR-C), $101.25\left(\mathrm{OCH}_{2} \mathrm{O}\right), 60.81$ (pyrazoline- $\mathrm{CH}$ ), 41.62 (pyrazoline- $\mathrm{CH}_{2}$ ), 34.14 (t-Butyl-C), 27.93 $\left(\mathrm{CH}_{3}\right)$. MS: for $\mathrm{C}_{22} \mathrm{H}_{23} \mathrm{~N}_{5} \mathrm{O}_{6}$, calculated. $453.16\left(\mathrm{M}^{+}+\mathrm{H}\right)$, found 454.15 .

\section{5-(benzo[d][1,3]dioxol-5-yl)-3-tert-butyl-N'-(3,4,5-trimethoxy- benzoyl)-4,5-dihydro-1 $H$-pyrazole-1-carbohydrazide (6)}

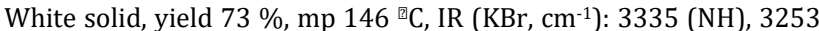
(NH), 1657 (broad, C=0, C=N). ${ }^{1} \mathrm{H}$ NMR $\left(\mathrm{CDCl}_{3}, 300 \mathrm{MHz}\right) \delta 9.31(\mathrm{~s}$, $1 \mathrm{H}, \mathrm{NHCO}$ ), 7.90 (d, $J=3.5 \mathrm{~Hz}, 1 \mathrm{H}, \mathrm{NHCO}$ ), 7.06 (s, 2H, AR-H), 6.83$6.68(\mathrm{~m}, 3 \mathrm{H}, \mathrm{AR}-\mathrm{H}), 5.99$ (dd, $\left.J=4.68,1.4 \mathrm{~Hz}, 2 \mathrm{H}, \mathrm{OCH}_{2} \mathrm{O}\right), 5.34$ (dd, $J$ $=11.6,4.7 \mathrm{~Hz}, 1 \mathrm{H}$, pyrazoline- $\mathrm{H}), 3.83\left(\mathrm{~s}, 3 \mathrm{H}, \mathrm{OCH}_{3}\right), 3.71(\mathrm{~s}, 6 \mathrm{H}$, $\left.\mathrm{OCH}_{3}\right), 3.43$ (dd, $J=17.9,11.7 \mathrm{~Hz}, 1 \mathrm{H}$, Pyrazoline-H), 2.80 (dd, $J=$ $17.8,4.8 \mathrm{~Hz}, 1 \mathrm{H}$, pyrazoline-H), $1.25\left(\mathrm{~s}, 9 \mathrm{H}, \mathrm{C}\left(\mathrm{CH}_{3}\right)_{3}\right) .{ }^{13} \mathrm{C} \mathrm{NMR}\left(\mathrm{CDCl}_{3}\right.$, $75 \mathrm{MHz}) \delta 166.2(\mathrm{C}=\mathrm{N}), 164.6(\mathrm{C}=0), 155.4(\mathrm{C}=0), 152.3$ (AR-C), 147.7 (AR-C), 146.2 (AR-C), 140.0 (AR-C), 131.5 (AR-C), 126.5 (ARC), 121.5 (AR-C), 107.7 (AR-C), 105.4 (AR-C), 103.8 (AR-C), 100.4 $\left(\mathrm{OCH}_{2} \mathrm{O}\right), 60.8\left(\mathrm{OCH}_{3}\right), 60.5\left(\mathrm{OCH}_{3}\right), 60.0$ (pyrazoline- $\left.\mathrm{CH}_{2}\right), 56.2$ $\left(\mathrm{OCH}_{3}\right), 42.1$ (pyrazoline-CH), 34.2(t-Butyl-C), $28.1\left(\mathrm{CH}_{3}\right)$. MS: for $\mathrm{C}_{25} \mathrm{H}_{30} \mathrm{~N}_{4} \mathrm{O}_{7}$, calculated calcd. $498.21\left(\mathrm{M}^{+}\right)$, found 498.54 .

5-(benzo[d][1,3]dioxol-5-yl)-3-tert-butyl-N'-(4-aminobenzoyl)4,5-dihydro-1H-pyrazole-1-carbohydrazide (7)

White solid, yield $86 \%, 188{ }^{\square} \mathrm{C}$, IR $\left(\mathrm{KBr}, \mathrm{cm}^{-1}\right): 3464\left(\mathrm{NH}_{2}\right), 3343$ $(\mathrm{NH}), 3235(\mathrm{NH}), 1633$ (broad, $\mathrm{C}=\mathrm{O}, \mathrm{C}=\mathrm{N}) .{ }^{1} \mathrm{H}$ NMR $\left(\mathrm{CDCl}_{3}, 300 \mathrm{MHz}\right)$ $\delta 8.33$ (s, 1H, NHCO), 7.96 (s, 1H, NHCO), 7.60 (d, $J=8.6 \mathrm{~Hz}, 2 \mathrm{H}, \mathrm{AR}-$ H) 6.79-6.60 (m, 3H, AR-H), $6.57(\mathrm{~d}, J=8.61 \mathrm{~Hz}, 2 \mathrm{H}, \mathrm{AR}-\mathrm{H}), 5.92(\mathrm{~s}$, $2 \mathrm{H}, \mathrm{OCH}_{2} \mathrm{O}$ ), 5.27 (dd, $J=11.7,5.0 \mathrm{~Hz}, 1 \mathrm{H}$, pyrazoline- $\left.\mathrm{H}\right), 3.48(\mathrm{~s}, 2 \mathrm{H}$,
$\mathrm{NH}_{2}$ ), 3.36 (dd, $J=17.9,11.7 \mathrm{~Hz}, 1 \mathrm{H}$, pyrazoline- $\mathrm{H}$ ), 2.75 (dd, $J=17.9$, $5.1 \mathrm{~Hz}, 1 \mathrm{H}$, pyrazoline-H), $1.23\left(\mathrm{~s}, 9 \mathrm{H}, \mathrm{C}\left(\mathrm{CH}_{3}\right)_{3}\right) .{ }^{13} \mathrm{C} \mathrm{NMR}\left(\mathrm{CDCl}_{3}, 75\right.$ $\mathrm{MHz}) \delta 166.3(\mathrm{C}=\mathrm{N}), 164.1(\mathrm{C}=0), 154.5(\mathrm{C}=0), 150.0$ (AR-C), 148.0 (AR-C), 147.1 (AR-C), 137.3 (AR-C), 129.2 (AR-C), 121.5 (AR-C), 119.1 (AR-C), 113.4 (AR-C), 109.4 (AR-C), 107.5 (AR-C), 100.5 $\left(\mathrm{OCH}_{2} \mathrm{O}\right), 60.5$ (pyrazoline- $\left.\mathrm{CH}_{2}\right), 42.2($ pyrazoline- $\mathrm{CH}), 33.8(\mathrm{t}-\mathrm{Butyl}-$ C), 28.5 $\left(\mathrm{CH}_{3}\right)$. MS: for $\mathrm{C}_{22} \mathrm{H}_{25} \mathrm{~N}_{5} \mathrm{O}_{4}$, calculated. $423.19\left(\mathrm{M}^{+}\right)$, found 423.20 .

5-(benzo[d][1,3]dioxol-5-yl)-3-tert-butyl-N'-(4-chlorobenzoyl)4,5-dihydro-1H-pyrazole-1-carbohydrazide (8)

White solid, yield 75\%, mp $152{ }^{\square} \mathrm{C}$, IR ( $\left.\mathrm{KBr}, \mathrm{cm}^{-1}\right): 3449(\mathrm{NH}), 3235$ $(\mathrm{NH}), 1643$ (broad, $\mathrm{C}=\mathrm{O}, \mathrm{C}=\mathrm{N}) .{ }^{1} \mathrm{H} \mathrm{NMR}\left(\mathrm{CDCl}_{3}, 300 \mathrm{MHz}\right) \delta 8.20(\mathrm{~d}, J=$ $4.3 \mathrm{~Hz}, 1 \mathrm{H}, \mathrm{NHCO}), 7.99$ (d, $J=4.3 \mathrm{~Hz}, 1 \mathrm{H}, \mathrm{NHCO}), 7.75$ (d, $J=8.66 \mathrm{~Hz}$, 2H, AR-H), 7.40 (d, $J=8.62 \mathrm{~Hz}, 2 \mathrm{H}, \mathrm{AR}-\mathrm{H}), 6.82-6.67$ (m, 3H, AR-H), $5.96\left(\mathrm{~s}, 2 \mathrm{H}, \mathrm{OCH}_{2} \mathrm{O}\right), 5.31(\mathrm{dd}, J=11.6,5.1 \mathrm{~Hz}, 1 \mathrm{H}$, pyrazoline- $\mathrm{H}), 3.44$ (dd, $J=17.93,11.6 \mathrm{~Hz}, 1 \mathrm{H}$, pyrazoline-H), 2.81 (dd, $J=17.9,5.1 \mathrm{~Hz}$, $1 \mathrm{H}$, pyrazoline-H), $1.26\left(\mathrm{~s}, 9 \mathrm{H}, \mathrm{C}\left(\mathrm{CH}_{3}\right)_{3}\right) \cdot{ }^{13} \mathrm{C} \mathrm{NMR}\left(\mathrm{CDCl}_{3}, 75 \mathrm{MHz}\right) \delta$ 166.2(C=N), 165.1(C=0), 155.0(C=0), 148.1 (AR-C), 147.2 (AR-C), 138.0 (AR-C), 136.5 (AR-C), 129.8 (AR-C), 128.8 (AR-C), 128.0 (ARC), 118.5 (AR-C), 108.2(AR-C), 105.0 (AR-C), 100.4 $\left(\mathrm{OCH}_{2} \mathrm{O}\right)$, 59.1 (pyrazoline- $\left.\mathrm{CH}_{2}\right), \quad 40.5 \quad$ (pyrazoline-CH), 32.5 (t-butyl-C), 26.5 $\left(\mathrm{CH}_{3}\right)$. MS: for $\mathrm{C}_{22} \mathrm{H}_{23} \mathrm{ClN}_{4} \mathrm{O}_{4}$, calcd. $442.14\left(\mathrm{M}^{+}\right)$, found 442.15 .

5-(benzo[d][1,3]dioxol-5-yl)-3-tert-butyl-N'-(4-methylbenzoyl)4,5-dihydro-1H-pyrazole-1-carbohydrazide (9)

White solid, yield 52\%, mp $148^{\text {घ }} \mathrm{C}$, IR $\left(\mathrm{KBr}, \mathrm{cm}^{-1}\right): 3437(\mathrm{NH}), 3245$ $(\mathrm{NH}), 1644$ (broad, $\mathrm{C}=0, \mathrm{C}=\mathrm{N}) .{ }^{1} \mathrm{H}$ NMR $\left(\mathrm{CDCl}_{3}, 300 \mathrm{MHz}\right) \delta 8.43(\mathrm{~d}, J=$ $4.2 \mathrm{~Hz}, 1 \mathrm{H}, \mathrm{NHCO}), 7.99(\mathrm{~d}, J=4.1 \mathrm{~Hz}, 1 \mathrm{H}, \mathrm{NHCO}), 7.70(\mathrm{~d}, J=8.25 \mathrm{~Hz}$, 2H, AR-H), 7.19 (d, $J=8.0 \mathrm{~Hz}, 2 \mathrm{H}, \mathrm{AR}-\mathrm{H}), 6.79-6.66$ (m, 3H, AR-H), $5.94\left(\mathrm{q}, J=1.5 \mathrm{~Hz}, 2 \mathrm{H}, \mathrm{OCH}_{2} \mathrm{O}\right), 5.29(\mathrm{dd}, J=11.7,5.1 \mathrm{~Hz}, 1 \mathrm{H}$, pyrazoline-H), 3.39 (dd, $J=17.9,11.7 \mathrm{~Hz}, 1 \mathrm{H}$, pyrazoline-H), 2.79 (dd, $J=17.9,5.1 \mathrm{~Hz}, 1 \mathrm{H}$, pyrazoline- $\mathrm{H}), 2.39\left(\mathrm{~s}, 3 \mathrm{H}, \mathrm{CH}_{3}\right), 1.25(\mathrm{~s}, 9 \mathrm{H}$ $\left.\mathrm{C}\left(\mathrm{CH}_{3}\right)_{3}\right) \cdot{ }^{13} \mathrm{C}$ NMR $\left(\mathrm{CDCl}_{3}, 75 \mathrm{MHz}\right) \delta 167.4(\mathrm{C}=\mathrm{N}), 165.0(\mathrm{C}=0), 154.5$ (C=0), 148.5 (AR-C), 147.3 (AR-C), 142.5(AR-C), 136.3 (AR-C), 129.0 (AR-C), 127.5 (AR-C), 119.1 (AR-C), 108.4 (AR-C), 106.0 (AR-C), 101.0 $\left(\mathrm{OCH}_{2} \mathrm{O}\right), 60.5$ (pyrazoline- $\left.\mathrm{CH}_{2}\right), 41.5$ (pyrazoline- $\left.\mathrm{CH}\right), 33.8(\mathrm{t}-$ butyl-C), 27.5 $\left(\mathrm{CH}_{3}\right), 21.7\left(\mathrm{CH}_{3}\right)$. MS: for $\mathrm{C}_{23} \mathrm{H}_{26} \mathrm{~N}_{4} \mathrm{O}_{4}$, calculated. 422.20 $\left(\mathrm{M}^{+}\right)$, found 422.35 .

\section{5-(benzo[d][1,3]dioxol-5-yl)-3-tert-butyl-N'-(4-methoxy-} benzoyl)-4,5-dihydro-1H-pyrazole-1-carbohydrazide (10)

White solid, yield 56\%, mp $18{ }^{\square} \mathrm{C}$, IR $\left(\mathrm{KBr}, \mathrm{cm}^{-1}\right): 3247(\mathrm{NH})$, 1644(broad, $\mathrm{C}=\mathrm{O}, \mathrm{C}=\mathrm{N}) \cdot{ }^{1} \mathrm{H}$ NMR $\left(\mathrm{CDCl}_{3}, 300 \mathrm{MHz}\right) \delta 8.01(\mathrm{~d}, J=4.5$ $\mathrm{Hz}, 1 \mathrm{H}, \mathrm{NHCO}), 7.86$ (d, $J=4.4 \mathrm{~Hz}, 1 \mathrm{H}, \mathrm{NHCO}), 7.80$ (d, $J=8.54 \mathrm{~Hz}, 2 \mathrm{H}$, AR-H), 6.94 (d, $J=8.7 \mathrm{~Hz}, 2 \mathrm{H}, \mathrm{AR}-\mathrm{H}), 6.83-6.68(\mathrm{~m}, 3 \mathrm{H}, \mathrm{AR}-\mathrm{H}), 5.95$ $\left(\mathrm{s}, 2 \mathrm{H}, \mathrm{OCH}_{2} \mathrm{O}\right), 5.31$ (dd, $J=11.6,5.1 \mathrm{~Hz}, 1 \mathrm{H}$, pyrazoline- $\left.\mathrm{H}\right), 3.87(\mathrm{~s}$, $3 \mathrm{H}, \mathrm{OCH}_{3}$ ), 3.57-3.34 (dd, $J=11.87,17.86 \mathrm{~Hz}, 1 \mathrm{H}$, pyrazoline- $\mathrm{H}$ ), 2.81 (dd, $J=17.9,5.2 \mathrm{~Hz}, 1 \mathrm{H}$, pyrazoline-H), $1.26\left(\mathrm{~s}, 9 \mathrm{H}, \mathrm{C}\left(\mathrm{CH}_{3}\right)_{3}\right) \cdot{ }^{13} \mathrm{C}$ NMR $\left(\mathrm{CDCl}_{3}, 75 \mathrm{MHz}\right) \delta 166.3(\mathrm{C}=\mathrm{N}), 164.7$ (C=0), 162.4 (C=0), 154.6 (ARC), 148.0 (AR-C), 147.0 (AR-C), 136.4 (AR-C),129.3 (AR-C), 124.3 (AR-C), 118.9 (AR-C), 113.7 (AR-C), 108.4 (AR-C), 106.0 (AR-C), $101.1\left(\mathrm{OCH}_{2} \mathrm{O}\right), 60.3$ (pyrazoline- $\left.\mathrm{CH}_{2}\right), 55.4\left(\mathrm{OCH}_{3}\right), 41.9$ (pyrazoline$\mathrm{CH}), \quad 34.1$ (t-butyl-C),28.0 $\left(\mathrm{CH}_{3}\right)$. MS: for $\mathrm{C}_{23} \mathrm{H}_{26} \mathrm{~N}_{4} \mathrm{O}_{5}$, calculated. $438.19\left(\mathrm{M}^{+}\right)$, found 438.24 .

General procedure for synthesis of 5 -(benzo[d][1,3]dioxol-5yl)-3-tert-butyl-N'-arylidene-4,5-dihydro-1H-pyrazole-1carbohydrazide (11-16)

Glacial acetic acid ( $1 \mathrm{ml})$ was added to a solution of $2(0.5 \mathrm{~g}, 0.0016$ $\mathrm{mol})$ and the appropriate aldehyde $(0.0016 \mathrm{~mol})$ in absolute ethanol $(30 \mathrm{ml})$. The reaction mixture was stirred under reflux for the appropriate time (TLC controlled). The solvent was evaporated under reduced pressure to afford the respective crude products 1116. The crude products were purified by column chromatography using silica gel and $\mathrm{CHCl}_{3} /$ ethyl acetate $5 / 1 \mathrm{v} / \mathrm{v}$ as mobile phase.

\section{5-(benzo[d][1,3]dioxol-5-yl)-3-tert-butyl-N'-benzylidene-4,5-} dihydro-1H-pyrazole-1-carbohydrazide (11)

White solid, yield $62 \%, 162$ घC. IR (KBr, cm-1): $3304(\mathrm{NH}), 1691$ $(\mathrm{C}=0), 1520(\mathrm{C}=\mathrm{N}) .{ }^{1} \mathrm{H}$ NMR $\left(\mathrm{CDCl}_{3}, 300 \mathrm{MHz}\right) \delta 9.23(\mathrm{~s}, 1 \mathrm{H}, \mathrm{CH}=\mathrm{N})$ 8.15 (s, 1H, NHCO), 7.72 (dd, J = 7.3, $2.5 \mathrm{~Hz}, 2 \mathrm{H}, \mathrm{AR}-\mathrm{H}), 7.43-7.33$ (m, $3 \mathrm{H}, \mathrm{AR}-\mathrm{H}), 6.81-6.68$ (m, 3H, AR-H), 5.95 (q, $\mathrm{J}=1.4 \mathrm{~Hz}, 2 \mathrm{H}, \mathrm{OCH}_{2} \mathrm{O}$ ), 
5.39 (dd, $J=11.7,5.1 \mathrm{~Hz}, 1 \mathrm{H}$, pyrazoline-H), 3.43 (dd, $J=17.9$, $11.7 \mathrm{~Hz}, 1 \mathrm{H}$, pyrazoline-H), $2.83(\mathrm{dd}, J=17.9,5.1 \mathrm{~Hz}, 1 \mathrm{H}$, pyrazoline-H), $1.27\left(\mathrm{~s}, 9 \mathrm{H}, \mathrm{C}\left(\mathrm{CH}_{3}\right)_{3}\right) \cdot{ }^{13} \mathrm{C}$ NMR $\left(\mathrm{CDCl}_{3}, 75 \mathrm{MHz}\right) \delta$ 164.4(C=N, pyrazoline), 151.1 ( $\mathrm{C}=0), 148.1$ (AR-C), 147.1 (AR-C), $144.2(\mathrm{C}=\mathrm{N}), 136.5$ (AR-C), 134.4 (AR-C), 129.5 (AR-C), 128.2 (AR-C), 127.3 (AR-C), 119.6 (AR-C), 108.4 (AR-C), 106.5 (ARC),101.3 $\left(\mathrm{OCH}_{2} \mathrm{O}\right), 59.9$ (pyrazoline- $\left.\mathrm{CH}_{2}\right), 42.1$ (pyrazoline- $\mathrm{CH}$ ), 33.8(t-butyl-C), 28.2( $\left.\mathrm{CH}_{3}\right)$. MS: for $\mathrm{C}_{22} \mathrm{H}_{24} \mathrm{~N}_{4} \mathrm{O}_{3}$, calculated calcd. $392.18\left(\mathrm{M}^{+}\right)$, found 392.64 .

\section{5-(benzo[d][1,3]dioxol-5-yl)-3-tert-butyl-N'-(4-bromo-} benzylidene)-4,5-dihydro-1 $H$-pyrazole-1-carbohydrazide (12)

White solid, yield $90 \%, \mathrm{mp}$ 136-138 घC. IR (KBr, cm-1): $3340(\mathrm{NH})$, 1689 (C=0), $1515(\mathrm{C}=\mathrm{N}) .{ }^{1} \mathrm{H}$ NMR $\left(\mathrm{CDCl}_{3}, 300 \mathrm{MHz}\right) \delta 9.25(\mathrm{~s}, 1 \mathrm{H}$, $\mathrm{CH}=\mathrm{N}), 8.12$ (s, 1H, NHCO), 7.59 (d, $J=8.47 \mathrm{~Hz}, 2 \mathrm{H}, \mathrm{AR}-\mathrm{H}), 7.51$ (d, $J=$ $8.55 \mathrm{~Hz}, 2 \mathrm{H}, \mathrm{AR}-\mathrm{H}), 6.74(\mathrm{~m}, 3 \mathrm{H}, \mathrm{AR}-\mathrm{H}), 5.95(\mathrm{q}, J=1.5 \mathrm{~Hz}, 2 \mathrm{H}$, $\mathrm{OCH}_{2} \mathrm{O}$ ), 5.38 (dd, $J=11.7,5.1 \mathrm{~Hz}, 1 \mathrm{H}$, pyrazoline- $\mathrm{H}$ ), 3.57-3.35 (dd, $J$ $=11.7,18.0 \mathrm{~Hz}, 1 \mathrm{H}$, pyrazoline- $\mathrm{H}), 2.83(\mathrm{dd}, J=18.0,5.1 \mathrm{~Hz}, 1 \mathrm{H}$, pyrazoline-H), $1.26\left(\mathrm{~s}, 9 \mathrm{H}, \mathrm{C}\left(\mathrm{CH}_{3}\right)_{3}\right) \cdot{ }^{13} \mathrm{C} \mathrm{NMR}\left(\mathrm{CDCl}_{3}, 75 \mathrm{MHz}\right) \delta$ 164.5(C=N, pyrazoline), 151.4(C=0), 148.0 (AR-C), 147.1 (AR-C), 143.5(C=N), 136.4 (AR-C), 134.4 (AR-C), 132.3 (AR-C), 128.5 (AR-C), 123.2 (AR-C), 118.5 (AR-C), 108.4 (AR-C), 105.5 (AR-C), 100.5 $\left(\mathrm{OCH}_{2} \mathrm{O}\right.$ ), 60.2 (pyrazoline- $\mathrm{CH}_{2}$ ), 42.4 (pyrazoline-CH), 34.1(t-butylC), 29.0 $\left(\mathrm{CH}_{3}\right)$. MS: for $\mathrm{C}_{22} \mathrm{H}_{23} \mathrm{BrN}_{4} \mathrm{O}_{3}$, calculated. $470.1\left(\mathrm{M}^{+}\right)$, found $470.34,472.35\left(\mathrm{M}^{+}+2\right)$.

\section{5-(benzo[d][1,3]dioxol-5-yl)-3-tert-butyl-N'-(4-nitrobenzylidene)-} 4,5-dihydro-1H-pyrazole-1-carbohydrazide (13)

Yellow solid, yield 70 \%, mp 196-198 ${ }^{\square} \mathrm{C} . \mathrm{IR}\left(\mathrm{KBr}, \mathrm{cm}^{-1}\right): 3341(\mathrm{NH})$, $1683(\mathrm{C}=0), 1515(\mathrm{C}=\mathrm{N}) .{ }^{1} \mathrm{H}$ NMR $\left(\mathrm{CDCl}_{3}, 300 \mathrm{MHz}\right) \delta 9.44(\mathrm{~s}, 1 \mathrm{H}$, $\mathrm{CH}=\mathrm{N}), 8.30$ (s, 1H, NHCO), $8.25(\mathrm{~d}, J=8.7 \mathrm{~Hz}, 2 \mathrm{H}, \mathrm{AR}-\mathrm{H}), 7.87(\mathrm{~d}, J=$ $8.5 \mathrm{~Hz}, 2 \mathrm{H}, \mathrm{AR}-\mathrm{H}), 6.82-6.66(\mathrm{~m}, 3 \mathrm{H}, \mathrm{AR}-\mathrm{H}), 5.96(\mathrm{~d}, J=2.1 \mathrm{~Hz}, 2 \mathrm{H}$, $\mathrm{OCH}_{2} \mathrm{O}$ ), 5.39 (dd, $J=11.7,5.0 \mathrm{~Hz}, 1 \mathrm{H}$, pyrazoline- $\mathrm{H}$ ), 3.45 (dd, $J=$ 18.0, $11.7 \mathrm{~Hz}, 1 \mathrm{H}$, pyrazoline-H), $2.86(\mathrm{dd}, J=17.9,5.1 \mathrm{~Hz}, 1 \mathrm{H}$, pyrazoline-H), $1.28\left(\mathrm{~s}, 9 \mathrm{H}, \mathrm{C}\left(\mathrm{CH}_{3}\right)_{3}\right) \cdot{ }^{13} \mathrm{C} \mathrm{NMR}\left(\mathrm{CDCl}_{3}, 75 \mathrm{MHz}\right) \delta$ 165.1 ( $\mathrm{C}=\mathrm{N}$, pyrazoline), 151.5(C=0), 148.0 (AR-C), 147.2 (AR-C), 142.0(C=N), 141.3 (AR-C), 136.5 (AR-C), 128.5 (AR-C), 124.9 (AR-C), 119.1 (AR-C), 108.9 (AR-C), 107.1 (AR-C), $101.5\left(\mathrm{OCH}_{2} \mathrm{O}\right), 60.1$ (pyrazoline- $\mathrm{CH}_{2}$ ), 41.8 (pyrazoline- $\mathrm{CH}$ ), 33.8(t-butyl-C), 28.2 $\left(\mathrm{CH}_{3}\right)$. MS: for $\mathrm{C}_{22} \mathrm{H}_{23} \mathrm{~N}_{5} \mathrm{O}_{5}$, calculated. $437.17\left(\mathrm{M}^{+}\right)$, found 437.39.

\section{5-(benzo[d][1,3]dioxol-5-yl)-3-tert-butyl-N'-(3,4,5- trimethoxybenzylidene)-4,5-dihydro-1 $H$-pyrazole-1- carbohydrazide (14)}

White solid, yield $53 \%$, mp 230-232 ${ }^{\square} \mathrm{C}$. IR (KBr, cm-1): $3241(\mathrm{NH}), 1662$ $(\mathrm{C}=0), 1577(\mathrm{C}=\mathrm{N}) \cdot{ }^{1} \mathrm{H}$ NMR $\left(\mathrm{CDCl}_{3}, 300 \mathrm{MHz}\right) \delta 9.20(\mathrm{~s}, 1 \mathrm{H}, \mathrm{CH}=\mathrm{N}), 8.10$ (s, 1H, NHCO), 6.96 (s, 2H, AR-H), 6.82-6.67 (m, 3H, AR-H), 5.94 (q, J= 1.5 $\mathrm{Hz}, 2 \mathrm{H}, \mathrm{OCH}_{2} \mathrm{O}$ ), 5.36 (dd, $J=11.7,5.1 \mathrm{~Hz}, 1 \mathrm{H}$, Pyrazoline-H), 3.89 (s, 9H, $\mathrm{OCH}_{3}$ ), 3.43 (dd, $J=17.9,11.7 \mathrm{~Hz}, 1 \mathrm{H}$, pyrazoline- $\mathrm{H}$ ), 2.83 (dd, $J=17.9,5.2$ $\mathrm{Hz}, 1 \mathrm{H}$, pyrazoline- $\mathrm{H}), 1.26\left(\mathrm{~s}, 9 \mathrm{H}, \mathrm{C}\left(\mathrm{CH}_{3}\right)_{3}\right) \cdot{ }^{13} \mathrm{C} \mathrm{NMR}\left(\mathrm{CDCl}_{3}, 75 \mathrm{MHz}\right) \delta$ 164.9(C=N, pyrazoline), 152.5(C=0), 150.8 (AR-C), 148.1 (AR-C), 147.1 (AR-C), 144.3(C=N), 139.1 (AR-C), 136.5 (AR-C), 129.9 (AR-C), 118.9 (AR-C), 108.9 (AR-C), 107.2 (AR-C), 104.9 (AR-C), 101.3 (OCH $\left.\mathrm{OC}_{2} \mathrm{O}\right), 61.1$ (pyrazoline-CH), $60.0\left(\mathrm{OCH}_{3}\right), 56.9\left(\mathrm{OCH}_{3}\right), 41.9\left(\right.$ pyrazoline- $\left.\mathrm{CH}_{2}\right), 33.5(\mathrm{t}-$ butyl-C), 28.1 $\left(\mathrm{CH}_{3}\right)$. MS: for $\mathrm{C}_{25} \mathrm{H}_{30} \mathrm{~N}_{4} \mathrm{O}_{6}$, calculated. $482.22\left(\mathrm{M}^{+}+1\right)$, found 483.41 .

\section{5-(benzo[d][1,3]dioxol-5-yl)-3-tert-butyl-N'-(4-methyl-} benzylidene)-4,5-dihydro-1 $H$-pyrazole-1-carbohydrazide (15)

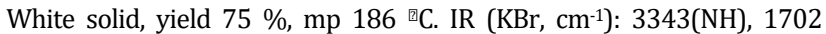
$(\mathrm{C}=0), 1501(\mathrm{C}=\mathrm{N}) .{ }^{1} \mathrm{H}$ NMR $\left(\mathrm{CDCl}_{3}, 300 \mathrm{MHz}\right) \delta 9.17(\mathrm{~s}, 1 \mathrm{H}, \mathrm{CH}=\mathrm{N}), 8.11$ (s, 1H, NHCO), 7.62 (d, $J=8.1 \mathrm{~Hz}, 2 \mathrm{H}, \mathrm{AR}-\mathrm{H}), 7.19$ (d, $J=7.9 \mathrm{~Hz}, 2 \mathrm{H}, \mathrm{AR}-\mathrm{H})$, 6.82-6.67 (m, 3H, AR-H), 5.95 (d, $J=1.5 \mathrm{~Hz}, 2 \mathrm{H}, \mathrm{OCH}_{2} \mathrm{O}$ ), 5.38 (dd, $J=$ $11.8,5.1 \mathrm{~Hz}, 1 \mathrm{H}$, pyrazoline- $\mathrm{H}), 3.42(\mathrm{dd}, J=17.9,11.7 \mathrm{~Hz}, 1 \mathrm{H}$, pyrazoline$\mathrm{H}), 2.82$ (dd, $J=17.9,5.1 \mathrm{~Hz}, 1 \mathrm{H}$, pyrazoline- $\mathrm{H}), 2.38\left(\mathrm{~s}, 3 \mathrm{H}, \mathrm{CH}_{3}\right), 1.26$ (s, 9H, $\left.\mathrm{C}\left(\mathrm{CH}_{3}\right)_{3}\right) .{ }^{13} \mathrm{C} \mathrm{NMR}\left(\mathrm{CDCl}_{3}, 75 \mathrm{MHz}\right) \delta 164.1(\mathrm{C}=\mathrm{N}$, pyrazoline), 151.2(C=0), 148.2 (AR-C), 147.1 (AR-C), 144.3(C=N), 139.8 (AR-C), 137.4 (AR-C), 132.3 (AR-C), 129.1 (AR-C), 126.9 (AR-C), 119.1 (AR-C), 109.1 (AR-C), 106.5 (AR-C), $101.5\left(\mathrm{OCH}_{2} \mathrm{O}\right), 60.5$ (pyrazoline-CH), 40.8 (pyrazoline- $\left.\mathrm{CH}_{2}\right), \quad 34.1$ (t-butyl-C), $28.5\left(\mathrm{CH}_{3}\right), 21.9\left(\mathrm{CH}_{3}\right)$. MS: for $\mathrm{C}_{23} \mathrm{H}_{26} \mathrm{~N}_{4} \mathrm{O}_{3}$, calculated. $406.2\left(\mathrm{M}^{+}\right)$, found 406.39 .
5-(benzo[d][1,3]dioxol-5-yl)-3-tert-butyl-N'-(4-methoxybenzylidene)-4,5-dihydro-1 $\mathrm{H}$-pyrazole-1-carbohydrazide (16)

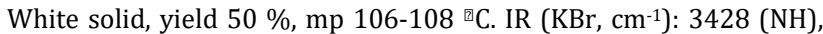
1689 (C=0), $1506(\mathrm{C}=\mathrm{N}) \cdot{ }^{1} \mathrm{H}$ NMR $\left(\mathrm{CDCl}_{3}, 300 \mathrm{MHz}\right) \delta 9.17(\mathrm{~s}, 1 \mathrm{H}$, $\mathrm{CH}=\mathrm{N}), 8.06(\mathrm{~s}, 1 \mathrm{H}, \mathrm{NHCO}), 7.64(\mathrm{~d}, J=8.1 \mathrm{~Hz}, 2 \mathrm{H}, \mathrm{AR}-\mathrm{H}), 6.88(\mathrm{~d}, J=$ $7.9 \mathrm{~Hz}, 2 \mathrm{H}, \mathrm{AR}-\mathrm{H}), 6.74-6.70(\mathrm{~m}, 3 \mathrm{H}, \mathrm{AR}-\mathrm{H}), 5.95(\mathrm{t}, J=1.86 \mathrm{~Hz}, 2 \mathrm{H}$, $\left.\mathrm{OCH}_{2} \mathrm{O}\right), 5.37$ (dd, $J=11.73,5.07 \mathrm{~Hz}, 1 \mathrm{H}$, pyrazoline-H), $3.83(\mathrm{~s}, 3 \mathrm{H}$, $\left.\mathrm{OCH}_{3}\right), 3.41$ (dd, $J=15.21,11.79 \mathrm{~Hz}, 1 \mathrm{H}$, pyrazoline- $\left.\mathrm{H}\right), 2.80(\mathrm{dd}, J=$ 13.89, $5.01 \mathrm{~Hz}, 1 \mathrm{H}$, pyrazoline-H), $1.20\left(\mathrm{~s}, 9 \mathrm{H}, \mathrm{C}\left(\mathrm{CH}_{3}\right)_{3}\right) \cdot{ }^{13} \mathrm{C}$ NMR $\left(\mathrm{CDCl}_{3}, 75 \mathrm{MHz}\right) \delta 164.9(\mathrm{C}=\mathrm{N}$, pyrazoline), 161.2(C=0), $151.3(\mathrm{AR}-\mathrm{C})$, 148.1 (AR-C), 146.9 (AR-C), 144.4(C=N), 136.5 (AR-C), 128.6 (AR-C) 127.2 (AR-C), 119.0 (AR-C), 113.9 (AR-C), 108.4 (AR-C), 106.0 (ARC), $\quad 101.0\left(\mathrm{OCH}_{2} \mathrm{O}\right), \quad 59.8$ (pyrazoline-CH), $55.3\left(\mathrm{OCH}_{3}\right), 41.8$ (pyrazoline- $\mathrm{CH}_{2}$ ), 34.0(t-butyl-C), 27.9 $\left(\mathrm{CH}_{3}\right)$. MS: for $\mathrm{C}_{23} \mathrm{H}_{26} \mathrm{~N}_{4} \mathrm{O}_{4}$, calculated. $422.2\left(\mathrm{M}^{+}\right)$, found 422.37 .

General procedure for synthesis of 2-(5-(benzo[d][1,3]dioxol-5yl)-3-tert-butyl-4,5-dihydro-1H-pyrazol-1-yl)-5-(aryl)-1,3,4oxadiazole17-22

A solution of an appropriate derivative 3-9in phosphorus oxychloride $\left(\mathrm{POCl}_{3}, 5.0 \mathrm{ml}\right)$ was refluxed for $3 \mathrm{~h}$, diluted carefully with water then $\mathrm{NaHCO}_{3}$ was added till being alkaline. The precipitate was filtered and recrystallized from ethanol to afford compounds 17-23.

\section{2-(5-(benzo[d][1,3]dioxol-5-yl)-3-tert-butyl-4,5-dihydro-1H-} pyrazol-1-yl)-5-phenyl-1,3,4-oxadiazole (17)

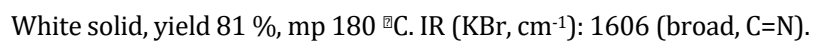

${ }^{1} \mathrm{H} \mathrm{NMR}\left(\mathrm{CDCl}_{3}, 300 \mathrm{MHz}\right) \delta 8.01-7.92(\mathrm{~m}, 2 \mathrm{H}, \mathrm{AR}-\mathrm{H}), 7.48(\mathrm{~d}, J=2.7$ Hz, 1H, AR-H), 7.46 (s, 2H, AR-H), 6.94-6.76 (m, 3H, AR-H), 5.97 (d, J $\left.=3.2 \mathrm{~Hz}, 2 \mathrm{H}, \mathrm{OCH}_{2} \mathrm{O}\right), 5.35$ (dd, $J=11.5,7.4 \mathrm{~Hz}, 1 \mathrm{H}$, Pyrazoline-H), 3.52 (dd, J = 18.04, 11.99 Hz, 1H, Pyrazoline-H), 2.96 (dd, $J=17.6,7.5$ $\mathrm{Hz}, 1 \mathrm{H}$, Pyrazoline-H), $1.31\left(\mathrm{~s}, 9 \mathrm{H}, \mathrm{C}\left(\mathrm{CH}_{3}\right)_{3}\right) .{ }^{13} \mathrm{C} \mathrm{NMR}\left(\mathrm{CDCl}_{3}, 75 \mathrm{MHz}\right)$ $\delta 166.0(\mathrm{C}=\mathrm{N}), 161.3(\mathrm{C}=\mathrm{N}), 160.4(\mathrm{C}=\mathrm{N}), 148.2(\mathrm{AR}-\mathrm{C}), 147.5(\mathrm{AR}-\mathrm{C})$, 134.6 (AR-C), 130.7 (AR-C), 128.8(AR-C), 126.1 (AR-C), 124.4 (ARC), 120.1 (AR-C), 108.5 (AR-C), 106.4 (AR-C), $101.2\left(\mathrm{OCH}_{2} \mathrm{O}\right), 63.8$ (Pyrazoline-CH), 43.0 (Pyrazoline- $\mathrm{CH}_{2}$ ), 34.2(t-Butyl-C), 28.1 $\left(\mathrm{CH}_{3}\right.$ ). MS: for $\mathrm{C}_{22} \mathrm{H}_{22} \mathrm{~N}_{4} \mathrm{O}_{3}$, calculated. $390.17\left(\mathrm{M}^{+}\right)$, found 390.19.

\section{2-(5-(benzo[d][1,3]dioxol-5-yl)-3-tert-butyl-4,5-dihydro-1H- pyrazol-1-yl)-5-(4-bromophenyl)-1,3,4-oxadiazole (18)}

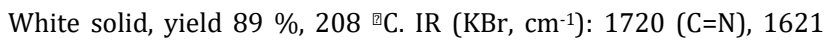
$(\mathrm{C}=\mathrm{N}) .{ }^{1} \mathrm{H}$ NMR $\left(\mathrm{CDCl}_{3}, 300 \mathrm{MHz}\right) \delta 7.83(\mathrm{~d}, J=8.3 \mathrm{~Hz}, 2 \mathrm{H}, \mathrm{AR}-\mathrm{H})$, $7.60(\mathrm{~d}, J=8.3 \mathrm{~Hz}, 2 \mathrm{H}, \mathrm{AR}-\mathrm{H}), 6.93-6.75(\mathrm{~m}, 3 \mathrm{H}, \mathrm{AR}-\mathrm{H}), 5.97(\mathrm{~d}, J=$ $1.5 \mathrm{~Hz}, 2 \mathrm{H}, \mathrm{OCH}_{2} \mathrm{O}$ ), 5.34 (dd, $J=11.5,7.4 \mathrm{~Hz}, 1 \mathrm{H}$, Pyrazoline- $\mathrm{H}$ ), 3.54 (dd, $J=17.7,11.5 \mathrm{~Hz}, 1 \mathrm{H}$, Pyrazoline-H), 2.96 (dd, $J=17.8,7.4 \mathrm{~Hz}, 1 \mathrm{H}$, Pyrazoline-H), $1.32\left(\mathrm{~s}, 9 \mathrm{H}, \mathrm{C}\left(\mathrm{CH}_{3}\right)_{3}\right) .{ }^{13} \mathrm{C}$ NMR $\left(\mathrm{CDCl}_{3}, 75 \mathrm{MHz}\right) \delta$ $164.1(\mathrm{C}=\mathrm{N}), 160.0(\mathrm{C}=\mathrm{N}), 158.1(\mathrm{C}=\mathrm{N}), 148.0(\mathrm{AR}-\mathrm{C}), 147.1(\mathrm{AR}-\mathrm{C})$, 134.3 (AR-C), 132.0(AR-C), 127.5(AR-C),125.2(AR-C), 123.5 (AR-C), 120.0(AR-C), 109.0(AR-C), 106.5 (AR-C), $101.5\left(\mathrm{OCH}_{2} \mathrm{O}\right), 63.3$ (Pyrazoline-CH), 43.1 (Pyrazoline- $\left.\mathrm{CH}_{2}\right), 33.8$ (t-Butyl-C), 28.2( $\mathrm{CH}_{3}$ ). MS: for $\mathrm{C}_{22} \mathrm{H}_{21} \mathrm{BrN}_{4} \mathrm{O}_{3}$, calculated. $468.08\left(\mathrm{M}^{+}\right)$, found 468.14 .

\section{2-(5-(benzo[d][1,3]dioxol-5-yl)-3-tert-butyl-4,5-dihydro-1 $H$ - pyrazol-1-yl)-5-(4-nitrophenyl)-1,3,4-oxadiazole (19)}

Greenish yellow solid, yield $97 \%$, mp 234 घC. IR (KBr, $\left.\mathrm{cm}^{-1}\right): 1620$ $(\mathrm{C}=\mathrm{N}) .{ }^{1} \mathrm{H}$ NMR $\left(\mathrm{CDCl}_{3}, 300 \mathrm{MHz}\right) \delta 8.34(\mathrm{~d}, J=8.96 \mathrm{~Hz}, 2 \mathrm{H}, \mathrm{AR}-\mathrm{H})$, $8.14(\mathrm{~d}, J=8.96 \mathrm{~Hz}, 2 \mathrm{H}, \mathrm{AR}-\mathrm{H}), 6.93-6.76(\mathrm{~m}, 3 \mathrm{H}, \mathrm{AR}-\mathrm{H}), 5.98(\mathrm{dd}, J=$ $1.49,3.88 \mathrm{~Hz}, 2 \mathrm{H}, \mathrm{OCH}_{2} \mathrm{O}$ ), $5.38(\mathrm{dd}, J=11.4,7.0 \mathrm{~Hz}, 1 \mathrm{H}$, PyrazolineH), 3.57 (dd, $J=17.9,11.5 \mathrm{~Hz}, 1 \mathrm{H}$, Pyrazoline-H), 3.00 (dd, $J=17.9$, $7.0 \mathrm{~Hz}, 1 \mathrm{H}$, Pyrazoline- $\mathrm{H}), 1.33\left(\mathrm{~s}, 9 \mathrm{H}, \mathrm{C}\left(\mathrm{CH}_{3}\right)_{3}\right) .{ }^{13} \mathrm{C}$ NMR $\left(\mathrm{CDCl}_{3}, 75\right.$ MHz) $\delta 167.1(\mathrm{C}=\mathrm{N}), 161.5(\mathrm{C}=\mathrm{N}), 158.5(\mathrm{C}=\mathrm{N}), 148.8(\mathrm{AR}-\mathrm{C}), 148.1$ (AR-C), 147.5 (AR-C), 134.3 (AR-C), 129.9 (AR-C), 127.2 (AR-C), 124.1 (AR-C), 120.0 (AR-C), 108.5 (AR-C), 106.3 (AR-C), $101.4\left(\mathrm{OCH}_{2} \mathrm{O}\right), 63.5$ (Pyrazoline-CH), 43.5 (Pyrazoline- $\left.\mathrm{CH}_{2}\right), 34.0$ (t-Butyl-C), 28.1 $\left(\mathrm{CH}_{3}\right)$. MS: for $\mathrm{C}_{22} \mathrm{H}_{21} \mathrm{~N}_{5} \mathrm{O}_{5}$, calculated. $435.15\left(\mathrm{M}^{+}\right)$, found 435.38 .

2-(5-(benzo[d][1,3]dioxol-5-yl)-3-tert-butyl-4,5-dihydro- $1 H$ pyrazol-1-yl)-5-(3,4,5-trimethoxyphenyl)-1,3,4-oxadiazole (20)

White solid, yield $75 \%$, IR (KBr, cm-1): $1715(\mathrm{C}=\mathrm{N}), 1617$ (C=N). ${ }^{1} \mathrm{H}$ NMR ( $\left.\mathrm{CDCl}_{3}, 300 \mathrm{MHz}\right) \delta 7.12(\mathrm{~s}, 2 \mathrm{H}, \mathrm{AR}-\mathrm{H}), 6.75-6.87$ (m, 3H,AR-H), 
$5.93\left(\mathrm{~s}, 2 \mathrm{H}, \mathrm{OCH}_{2} \mathrm{O}\right), 5.35(\mathrm{~m}, 1 \mathrm{H}$, Pyrazoline- $\mathrm{H}), 3.89(\mathrm{~s}, 9 \mathrm{H}$, $\left.\left(\mathrm{OCH}_{3}\right)_{3}\right), 3.48-3.54(\mathrm{~m}, 1 \mathrm{H}$, Pyrazoline-H), $2.93(\mathrm{~d}, J=13.16 \mathrm{~Hz}, 1 \mathrm{H}$, Pyrazoline-H), 1.29 (s, 9H, C( $\left.\left.\mathrm{CH}_{3}\right)_{3}\right)$. MS: for $\mathrm{C}_{25} \mathrm{H}_{28} \mathrm{~N}_{4} \mathrm{O}_{6}$, calcd. $480.20\left(\mathrm{M}^{+}\right)$, found 480.28 .

\section{2-(5-(benzo[d][1,3]dioxol-5-yl)-3-tert-butyl-4,5-dihydro-1H- pyrazol-1-yl)-5-(4-aminophenyl)-1,3,4-oxadiazole (21)}

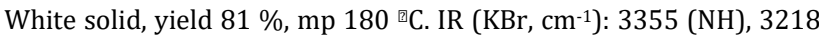
$(\mathrm{NH}), 1700(\mathrm{C}=\mathrm{N}), 1607$ (broad, $\mathrm{C}=\mathrm{N}) .{ }^{1} \mathrm{H} \mathrm{NMR}\left(\mathrm{CDCl}_{3}, 300 \mathrm{MHz}\right) \delta$ $7.74(\mathrm{~d}, J=8.65 \mathrm{~Hz}, 2 \mathrm{H}, \mathrm{AR}-\mathrm{H}), 6.93-6.73(\mathrm{~m}, 3 \mathrm{H}, \mathrm{AR}-\mathrm{H}), 6.70(\mathrm{~d}, J=$ $8.65 \mathrm{~Hz}, 2 \mathrm{H}, \mathrm{AR}-\mathrm{H}), 5.96\left(\mathrm{q}, J=1.42 \mathrm{~Hz}, 2 \mathrm{H}, \mathrm{OCH}_{2} \mathrm{O}\right), 5.39-5.24(\mathrm{~m}$, $1 \mathrm{H}$, Pyrazoline- $\mathrm{H}), 3.58-3.42\left(\mathrm{~m}, 3 \mathrm{H}\right.$, Pyrazoline- $\mathrm{H}$ and $\left.\mathrm{NH}_{2}\right), 2.93$ (dd, $J=17.7,7.8 \mathrm{~Hz}, 1 \mathrm{H}$, Pyrazoline- $\mathrm{H}), 1.30\left(\mathrm{~s}, 9 \mathrm{H}, \mathrm{C}\left(\mathrm{CH}_{3}\right)_{3}\right) .{ }^{13} \mathrm{C} \mathrm{NMR}$ $\left(\mathrm{CDCl}_{3}, 75 \mathrm{MHz}\right) \delta 165.49(\mathrm{C}=\mathrm{N}), 160.91(\mathrm{C}=\mathrm{N}), 160.78(\mathrm{C}=\mathrm{N}), 148.73$ (AR-C), 148.11 (AR-C), 147.36 (AR-C), 134.64 (AR-C), 127.83 (AR-C), 120.11 (AR-C), 114.64 (AR-C), 114.42 (AR-C), 108.42 (AR-C), 106.50 (AR-C), $101.15\left(\mathrm{OCH}_{2} \mathrm{O}\right), 63.91$ (Pyrazoline- $\left.\mathrm{CH}\right), 42.96$ (Pyrazoline$\mathrm{CH}_{2}$ ), 34.18 (t-Butyl-C), 28.12( $\left(\mathrm{CH}_{3}\right)$. MS: for $\mathrm{C}_{22} \mathrm{H}_{23} \mathrm{~N}_{5} \mathrm{O}_{3}$, calculated. $405.18\left(\mathrm{M}^{+}\right)$, found 405.34 .

\section{2-(5-(benzo[d][1,3]dioxol-5-yl)-3-tert-butyl-4,5-dihydro-1H- pyrazol-1-yl)-5-(4-chlorophenyl)-1,3,4-oxadiazole (22)}

Yellow solid, yield $86 \%$, mp $200{ }^{\circledR} \mathrm{C}$. IR $\left(\mathrm{KBr}, \mathrm{cm}^{-1}\right): 1718$ (C=N), 1619 $(\mathrm{C}=\mathrm{N}) .{ }^{1} \mathrm{H}$ NMR $\left(\mathrm{CDCl}_{3}, 300 \mathrm{MHz}\right) \delta 7.80(\mathrm{~s}, 2 \mathrm{H}, \mathrm{AR}-\mathrm{H}), 7.32-7.43(\mathrm{~s}$, 2H,AR-H), 6.70-6.87 (m, 3H,AR-H), 5.95 (s, 2H, OCH $\mathrm{OC}_{2}$ ), 5.37 (brs, $1 \mathrm{H}$, Pyrazoline-H), 3.56 (m, 1H, Pyrazoline- $\mathrm{H}), 2.95$ (m, 1H, Pyrazoline- $\mathrm{H})$, $1.30\left(\mathrm{~s}, 9 \mathrm{H}, \mathrm{C}\left(\mathrm{CH}_{3}\right)_{3}\right) \cdot{ }^{13} \mathrm{C}$ NMR $\left(\mathrm{CDCl}_{3}, 75 \mathrm{MHz}\right) \delta 167.63(\mathrm{C}=\mathrm{N})$, 160.11(C=N), 159.17(C=N), 148.24 (AR-C), 147.61 (AR-C), 137.09 (ARC), 133.88 (AR-C), 129.55 (AR-C), 127.49 (AR-C), 122.34 (AR-C), 120.25 (AR-C), 108.53 (AR-C), 106.29 (AR-C), $101.25\left(\mathrm{OCH}_{2} \mathrm{O}\right), 63.68$ (Pyrazoline-CH), 43.23 (Pyrazoline- $\mathrm{CH}_{2}$ ), 34.34(t-Butyl-C), $28.09\left(\mathrm{CH}_{3}\right)$. MS: for $\mathrm{C}_{22} \mathrm{H}_{21} \mathrm{ClN}_{4} \mathrm{O}_{3}$, calculated. $424.13\left(\mathrm{M}^{+}\right)$, found 424.18.

\section{2-(5-(benzo[d][1,3]dioxol-5-yl)-3-tert-butyl-4,5-dihydro-1H- pyrazol-1-yl)-5-p-tolyl-1,3,4-oxadiazole (23)}

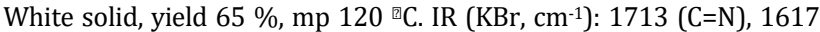
$(\mathrm{C}=\mathrm{N}) .{ }^{1} \mathrm{H}$ NMR $\left(\mathrm{CDCl}_{3}, 300 \mathrm{MHz}\right) \delta 7.85(\mathrm{~d}, J=8.15 \mathrm{~Hz}, 2 \mathrm{H}, \mathrm{AR}-\mathrm{H}), 7.27$ (d, $J=8.0 \mathrm{~Hz}, 2 \mathrm{H}, \mathrm{AR}-\mathrm{H}), 6.94-6.75(\mathrm{~m}, 3 \mathrm{H}, \mathrm{AR}-\mathrm{H}), 5.97$ (dd, $J=1.51$, $3.79 \mathrm{~Hz}, 2 \mathrm{H}, \mathrm{OCH}_{2} \mathrm{O}$ ), 5.33 (dd, $J=7.8,11.3 \mathrm{~Hz}, 1 \mathrm{H}$, Pyrazoline-H), 3.52 (dd, $J=17.7,11.4 \mathrm{~Hz}, 1 \mathrm{H}$, Pyrazoline-H), 2.95 (dd, $J=17.7,7.6 \mathrm{~Hz}, 1 \mathrm{H}$, Pyrazoline- $\mathrm{H}), 2.42\left(\mathrm{~s}, 3 \mathrm{H}, \mathrm{CH}_{3}\right), 1.32\left(\mathrm{~s}, 9 \mathrm{H}, \mathrm{C}\left(\mathrm{CH}_{3}\right)_{3}\right) .{ }^{13} \mathrm{C} \mathrm{NMR}\left(\mathrm{CDCl}_{3}\right.$, $75 \mathrm{MHz}) \delta 165.81(\mathrm{C}=\mathrm{N}), 161.09(\mathrm{C}=\mathrm{N}), 160.55(\mathrm{C}=\mathrm{N}), 148.16(\mathrm{AR}-\mathrm{C})$ 147.42(AR-C), 140.95(AR-C), 134.53(AR-C), 129.45(AR-C), 126.11(ARC), 121.63(AR-C), 120.08(AR-C), 108.44 (AR-C), 106.43(AR-C), 101.18 $\left(\mathrm{OCH}_{2} \mathrm{O}\right), \quad$ 63.83(Pyrazoline- $\left.\mathrm{CH}\right), \quad$ 43.01(Pyrazoline- $\left.\mathrm{CH}_{2}\right)$, 34.21(t-Butyl-C), 28.12( $\left(\mathrm{CH}_{3}\right), \quad 21.32\left(\mathrm{CH}_{3}\right)$. MS: for $\mathrm{C}_{23} \mathrm{H}_{24} \mathrm{~N}_{4} \mathrm{O}_{3}$, calculated. $404.18\left(\mathrm{M}^{+}\right)$, found 404.31 .

\section{Biological studies}

Animals

Adult male Swiss albino mice (weighing 18-25 g) used in this study were purchased from the Animal House Colony of the National Research Centre, Cairo, Egypt. Animals were housed under standardized conditions (room temperature $23+2{ }^{\circ} \mathrm{C}$; relative humidity 55+5\%;12 h-light/dark cycle) and had free access to tap water and standard rat chow throughout the whole experimental period. All animal procedures were performed after receiving approval from the Ethics Committee of the National Research Center Reg no 15-190, in accordance with the recommendations for the proper care and use of laboratory animals "Canadian Council on Animal Care Guidelines, 1984". After seven days of adaptation to laboratory conditions, the animals were randomly assigned to control and experimental groups consisting of 6 mice each.

\section{Drugs and chemicals}

All test compounds were suspended in $7 \%$ tween- 80 and administered intraperitoneally at a volume of $0.1 \mathrm{ml} / 10 \mathrm{~g}$ body weight. Tween-80 and pentylenetetrazole were purchased from Sigma, St. Louis, HO, USA. Meanwhile, stiripentol (STP) was synthesized according to the reported procedure [8].

\section{Methods}

\section{Subcutaneous pentylenetetrazole (scPTZ) screen}

This test produces threshold or minimal (clonic) seizures. An aqueous solution of PTZ at a dose of $85 \mathrm{mg} / \mathrm{kg}$ was administered subcutaneously (sc) in the loose fold of skin at the back of the mice neck. This dose, denoted convulsive dose $97\left(\mathrm{CD}_{97}\right)$, causes seizures in more than $97 \%$ of animals. The test is carried out by giving $s c \mathrm{PTZ}$, half an hour post-injection of the test compounds (i. p.). The tested animals were closely monitored for the occurrence of seizuresfor 30 min. A threshold convulsion was defined as one episode of clonic convulsions which persisted for at least a 5 seconds period. The absence of a single 5-second episode of clonic spasms during the period of observation is taken as the end point in this test [14,15].

\section{Maximal electroshock seizure (MES) screen}

Electroconvulsions were induced, half an hour after the intraperitoneal injection of the test compounds, by a current (fixed current intensity of $25 \mathrm{~mA}, 0.2 \mathrm{~s}$ stimulus duration) delivered via ear clip electrodes using a Rodent Shocker generator (constant-current stimulator Type 221, Hugo Sachs Elektronik, Freiburg, Germany). The criterion for the occurrence of seizure activity was the tonic hind limb extension (i.e., the hind limbs of animals outstretched 180 ${ }^{\circ}$ to the plane of the body axis) [16].

\section{Neurotoxicity}

The neurotoxicity of the animals was evaluated by adopting the rotarod test [17]. In this test, the animals were trained to maintain equilibrium on a rotating 1-inch diameter knurled plastic rod (rotarod, UGO Basile, 47600, Varese, Italy) at a speed of $10 \mathrm{rpm}$ for 60 seconds (sec) in each of three trials. Only animals that fulfil this criterion were included in the experiment. The animals in the experimental groups $(n=6)$ were given an i. p. injection of one of the test compound. Thirty minutes later, the mice were placed again on the rotating rod and the motor performance time was recorded up to $60 \mathrm{sec}$. The neurotoxicity was indicated by the inability of the animal to maintain equilibrium on the rod for at least $60 \mathrm{sec}$.

\section{RESULTS AND DISCUSSION}

\section{Chemistry}

Synthesis of compounds 3-22 and their starts 1 and 2 are shown in scheme 1.
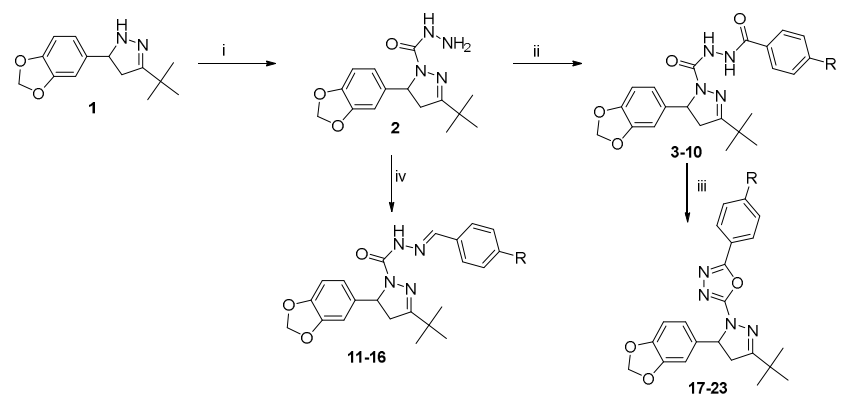

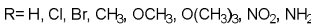

i) $\mathrm{ClCOCl}_{1} \mathrm{CHCl}_{3}, 10 \mathrm{~min}$, It then hydrazine hydrate; ii) appropriate acid chloride, $\mathrm{CHCl}_{3}$, triethylamine, rt: iii) POCb reflux, iv) appropriate aldehyde, absolute ethanol, glacial acetic acid, reflux.

Scheme 1: General method for the synthesis of compounds 2-22

Initially, 3,5-disubstituted 2-pyrazoline (compound 1) has been prepared as previously reported [8]. Then, the free base 1 has been converted, while fresh, to the main carbohydrazide derivative 2 . Indeed, compound 2 could be obtained from 1 via diverse chemical pathways like hydrazinolysis of the carbamoyl ester of compound 1 , resulting from the reaction of 1 with alkyl chloroformates. Moreover, the reaction of 1 with phosgene, followed by addition of hydrazine to the produced carbamoyl chloride will afford compound 2 in twostep one-pot reaction instead of two isolated reactions in the former method. In addition, the reaction of the carbazide with stiripentol starting chalcone (1-(benzo[d][1,3]dioxol-5-yl)-4,4-dimethylpent-1- 
en-3-one) will provide compound 2,however,it is not preferred due to the possibility of dimerization (scheme 2).

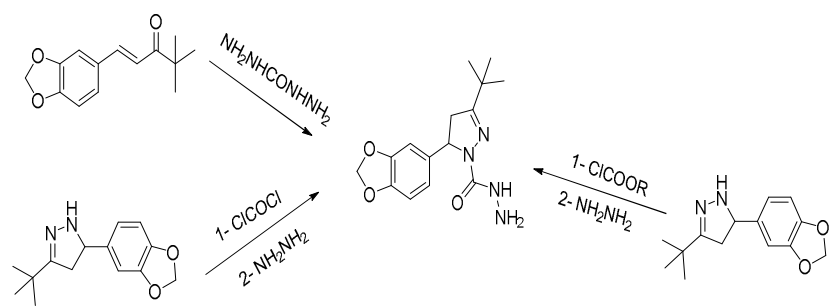

Scheme 2: Different synthetic pathways towards key compound 2

In the present study, it was preferred to apply phosgene method due to the availability of starting materials, in addition to being a short and non-drastic reaction. Compound 2 has been confirmed by IR and NMR. The IR displayed amidic $\mathrm{C}=0$ band at $1736 \mathrm{~cm}^{-1}$ and the $\mathrm{C}=\mathrm{N}$ at $1690 \mathrm{~cm}^{-1}$ beside the well-defined bands for NHs at 3451 and 3354 $\mathrm{cm}^{-1}$. For NMR, ${ }^{13} \mathrm{C}$ showed the carbonyl signal at $\delta 156.9 \mathrm{ppm}$, while the $\mathrm{C}=\mathrm{N}$ of 2 -pyrazoline appeared at $163.8 \mathrm{ppm}$. Further reaction of 2 with different acid chlorides at room temperature in chloroform and in presence of triethylamine generated series $\mathrm{A}$.

Compounds 3-10 have been characterized by IR, NMR, and mass spectroscopy as shown in the experimental section and supporting information. No major change was observed in the IR except the broadband at $1650 \mathrm{~cm}^{-1}$ for both carbonyl groups. In ${ }^{13} \mathrm{C}-\mathrm{NMR}$, a new carbonyl signal has been observed at $\delta 160-164$ ppm beside the old one at $\delta 153-157 \mathrm{ppm}$. The ${ }^{1} \mathrm{H}-\mathrm{NMR}$ showed more aromatic protons due to the aroyl moiety and the characteristic two doublet of doublet signals at 2.8 and $3.4 \mathrm{ppm}$ corresponding to the prochiral $\mathrm{CH}_{2}$ protons of 2-pyrazoline while $\mathrm{CH}$ proton showed triplet signal at 5.3 ppm. In addition, two singlet signals were found at 1.2 and $5.9 \mathrm{ppm}$ for $t$-butyl and the ethereal methylene bridge protons, respectively.
The most characteristic signals of this series are the two singlet signals of the amidic NHs at $\delta 8.0-9.0 \mathrm{ppm}$. The mass spectra for such series showed the molecular ion signal as the minor peak but the free 2-pyrazoline (1) signal $\left(\left(\mathrm{M}^{+}\right) 246\right.$ or $\left.\left(\mathrm{M}^{+}+1\right) 247\right)$ was present in all spectra as the base peak. The signal for the carbohydrazide intermediate (2) was rarely reported.

Series B has been prepared by the well-documented semicarbazone synthesis via reacting appropriate aldehyde with the key carbohydrazide (2) in ethanol in the presence of acetic acid. Compounds 11-16 have been identified by spectral analysis, where IR disclosed only one $\mathrm{NH}$ band at $3200-3450 \mathrm{~cm}^{-1}$, while the amidic $\mathrm{C}=\mathrm{O}$ and the imine $(\mathrm{C}=\mathrm{N})$ bands were displayed at 1650-1710 and $1500-1570 \mathrm{~cm}^{-1}$, respectively. In ${ }^{1} \mathrm{H}-\mathrm{NMR}$, only one amidic $\mathrm{NH}$ has been demonstrated at $\delta 8.0-8.30 \mathrm{ppm}$, whereas the most distinguishing signal of the semicarbazono-imine proton $(\mathrm{CH}=\mathrm{N})$ was displayed at $\delta 9.0-9.50 \mathrm{ppm} .{ }^{13} \mathrm{C}-\mathrm{NMR}$ for this series exhibited only one amidic $\mathrm{C}=0$ signal at $\delta$ 151-155 ppm, while the semicarbazono-imine carbon has been observed at $\delta 141-145 \mathrm{ppm}$. In the mass analysis, the 2-pyrazoline (1) still denotes intense signals, while the molecular ions exposed minor signals. 1,3,4Oxadiazole derivatives 17-22 (series C) have been achieved by means of intramolecular cyclodehydration of series A. Cyclodehydration is attainable under several conditions, for instance, heating in solvents such as pyridine, DMF, or in the presence of additives such as 1-ethyl-3-(3-dimethylaminopropyl) carbodiimide (EDC). $\mathrm{SOCl}_{2}, \mathrm{P}_{2} \mathrm{O}_{5}, \mathrm{H}_{2} \mathrm{SO}_{4}, \mathrm{POCl}_{3}$, Burgess reagent, triphenylphospine, and triflic anhydride are used for the cyclization [18]. In this study, $\mathrm{POCl}_{3}$ was used as a solvent and cyclodehydrating agent. Breaking down of $\mathrm{POCl}_{3}$ by the addition of water (very carefully and slowly) without cooling was a crucial step in order to get filterable powder. Spectral confirmation of compounds 17-22 has been performed, where the NH bands have fully disappeared in IR. In ${ }^{1} \mathrm{H}-\mathrm{NMR}$, no amidic NH has been reported, however, three imine signals at $\delta$ 158-166 ppm were revealedin ${ }^{13} \mathrm{C}-\mathrm{NMR}$. Compounds $17-22$ showed very high stability in the mass analysis, since in most of the compounds, such asand22, the molecular ion signal was the base peak.

Table 1: Anticonvulsant activity and neurotoxicity of compounds 3-22

\begin{tabular}{|c|c|c|c|c|c|c|}
\hline \multirow[t]{2}{*}{ Compound number } & \multirow[t]{2}{*}{$\mathbf{R}$} & \multicolumn{2}{|l|}{ dose } & \multicolumn{2}{|c|}{$\%$ protection } & \multirow[t]{2}{*}{ neurotoxicityc } \\
\hline & & $\mathrm{mg} / \mathrm{kg}$ & $\mu \mathrm{mol} / \mathrm{kg}$ & scPTZ $^{a}$ & $\mathbf{M E S}^{\mathbf{b}}$ & \\
\hline 3 & $\mathrm{H}$ & 125 & 306 & 83 & 50 & $0 / 6$ \\
\hline 4 & $\mathrm{Br}$ & 125 & 257 & 33 & 66 & $0 / 6$ \\
\hline 5 & $\mathrm{NO}_{2}$ & 125 & 275 & 100 & 83 & $0 / 6$ \\
\hline 6 & $3,4,5-\mathrm{OCH}_{3}$ & 125 & 251 & 66 & 0 & $0 / 6$ \\
\hline 7 & $\mathrm{NH}_{2}$ & 125 & 295 & 66 & 33 & $0 / 6$ \\
\hline 8 & $\mathrm{Cl}$ & 125 & 282 & 50 & 83 & $0 / 6$ \\
\hline 9 & $\mathrm{CH}_{3}$ & 125 & 296 & 50 & 66 & $0 / 6$ \\
\hline 10 & $\mathrm{OCH}_{3}$ & 125 & 285 & 50 & 0 & $0 / 6$ \\
\hline 11 & $\mathrm{H}$ & 100 & 255 & 50 & $\mathrm{X}$ & $\mathrm{X}$ \\
\hline 12 & $\mathrm{Br}$ & 100 & 212 & 83 & 66 & $1 / 6$ \\
\hline 13 & $\mathrm{NO}_{2}$ & 100 & 228 & 100 & 33 & $1 / 6$ \\
\hline 14 & $3,4,5-\mathrm{OCH}_{3}$ & 100 & 207 & 83 & 17 & $0 / 6$ \\
\hline 15 & $\mathrm{CH}_{3}$ & 100 & 246 & 83 & 0 & $1 / 6$ \\
\hline 16 & $\mathrm{OCH}_{3}$ & 100 & 236 & 83 & 17 & $0 / 6$ \\
\hline 17 & $\mathrm{H}$ & 125 & 320 & 50 & 17 & $0 / 6$ \\
\hline 18 & $\mathrm{Br}$ & 125 & 267 & 83 & 0 & $0 / 6$ \\
\hline 19 & $\mathrm{NO}_{2}$ & 125 & 287 & 100 & 33 & $0 / 6$ \\
\hline 20 & $3,4,5-\mathrm{OCH}_{3}$ & 125 & 260 & 50 & 0 & $0 / 6$ \\
\hline 21 & $\mathrm{NH}_{2}$ & 125 & 308 & 100 & 17 & $0 / 6$ \\
\hline 22 & $\mathrm{Cl}$ & 125 & 294 & 66 & 0 & $0 / 6$ \\
\hline 23 & $\mathrm{CH}_{3}$ & - & - & $\mathrm{X}$ & $\mathrm{X}$ & $\mathrm{X}$ \\
\hline Stiripentol $(\mathrm{I})^{\mathrm{d}}$ & - & 175 & 747 & 100 & $\mathrm{X}$ & $\mathrm{X}$ \\
\hline Stiripentol (I) ${ }^{\mathrm{d}}$ & - & 150 & 640 & 83 & 66 & $\mathrm{X}$ \\
\hline Stiripentol (I) ${ }^{\mathrm{d}}$ & - & 125 & 534 & 66 & $\mathrm{X}$ & $\mathrm{X}$ \\
\hline Stiripentol (I) & - & 115 & 491 & 50 & $\mathrm{X}$ & $X$ \\
\hline Stiripentol (I) ${ }^{\mathrm{d}}$ & - & 100 & 427 & 33 & $X$ & $\mathrm{X}$ \\
\hline Stiripentol (I) ${ }^{\mathrm{d}}$ & - & 90 & 384 & 17 & $\mathrm{X}$ & $\mathrm{X}$ \\
\hline $\mathrm{II}^{\mathrm{d}}$ & - & 100 & 346 & 50 & 100 & $8 / 8$ \\
\hline III $^{\mathrm{d}}$ & - & 150 & 350 & 100 & 17 & $0 / 8$ \\
\hline
\end{tabular}

Doses were administered i. p. Animals $(n=6)$ were examined at $0.5 \mathrm{~h}$ after administration of the test compounds. $\mathrm{X}$ indicates the compound not tested. aSubcutaneous pentylenetetrazole test. bMaximal electroshock test. cNeurotoxicity screening by rotorod test (number of animals exhibiting neurotoxicity/number of animals tested). ddata from [8]. 


\section{Pharmacology}

The predictable animal seizure models are the most widely used techniques for the preclinical discovery of novel bioactive chemical agents for the treatment of epilepsy. These animal seizure models cover diverse classes of epilepsy with different categories [3]. The acute seizure model, induced by either electric (MES) or chemical ( $s c$ PTZ) stimulus in normal animals, also known as the "gold standards", is a mechanism-independent most potent model for early stages of anticonvulsant testing. The anticonvulsant drug development (ADD) program protocol of National Institute of Health, provided by the epilepsy section of National Institute of Neurological Disorders and Stroke (NINIDS), has been followed in the current investigation.

The MES test uses electrical stimulus to produce generalized tonicclonic seizures (grand mal epilepsy) and identifies compounds that prevent seizure spreading. This experimental model simulates human tonic-clonic epilepsy and partial convulsions, with or without secondary generalization. The $s c$ PTZ screening test utilizes chemical stimuli to induce myoclonic seizures, simulating human generalized absence seizures (petit mal epilepsy) $[14,19,20]$. Target compounds 3-22 have been screened in the MES and $s c$ PTZ tests where candidates that elevated seizure threshold have been identified and selected. Furthermore, acute neurological toxicity was determined by the rotarod test.

New candidates 3-22 were administered intraperitoneally (ip) to male albino mice at a fixed dose of $125 \mathrm{mg} / \mathrm{kg}$ for series $\mathrm{A}$ and $\mathrm{C}$ and $100 \mathrm{mg} / \mathrm{kg}$ for series Bto evaluate anticonvulsant activity. These doses were selected according to a pilot study performed prior to the main study. The results of the primary (phase-I) screening are summarized in table 1 .

All candidates were active in both models of epilepsy except compounds $6,10,15,18,20$ and 22 , which have shown activity solely in $s c$ PTZ model. All compounds 3-22 were active against generalized absence seizures (petit mal epilepsy) related screen and have effectively increased the seizures threshold in scPTZ test. Compounds $5,13,19$, and 21 were the most active congeners displaying $100 \%$ protection at $275,228,287$, and $308 \mu \mathrm{mol} / \mathrm{kg}$, respectively and were $2.7,3.3,2.6$, and 2.8 folds more active than the reference compound stiripentol (I). On the other hand, Compounds 3, 12, 14, 15, 16 and 18 exhibited pronounced anticonvulsant activity as seizure threshold rises by protecting $83 \%$ of tested animals at a dose ranging from 207-
$308 \mu \mathrm{mol} / \mathrm{kg}$ and were 2-3 times more active than Stiripentol (640 $\mu \mathrm{mol} / \mathrm{kg}, 83 \%$ protection). Other compounds disclosed moderate activity showing $33-66 \%$ protection.

These candidates were less effective against seizure spreading of generalized tonic-clonic seizures (grand mal) screen. Thus in MES test, compounds 5 and 8 were the most effective surrogates showing $83 \%$ protection at 275 and $282 \mu \mathrm{mol} / \mathrm{kg}$, respectively. Interestingly, compounds 5 and 8 were more potent than stiripentol, which showed $66 \%$ maximal protection at $640 \mu \mathrm{mol} / \mathrm{kg}$. Compounds 4, 9, and 12 were $2.5,2.1$ and 3.0 times stronger than the reference drug (I) showing $66 \%$ protection at 257, 296, and $212 \mu \mathrm{mol} / \mathrm{kg}$, respectively. Regarding neurotoxicity, the new candidates showed greater safety profile, since all compounds caused no motor impairment in the rotarod test. The only exceptions were compounds 12,13 , and 15 , which produced slight motor impairment in only 1 out of 6 mice.

In comparison to lead compounds II and III, which have been used in the rational ligand based design of this study, compounds 3-22 have exhibited more potent and broader spectrum anticonvulsant profile. Compounds 5, 13, 19, and 21 were 1.27, 1.5, 1.2, and 1.13 folds more active than lead compound III in scPTZ screen, respectively. Noteworthy, they displayed superior activity to compound III against MES screen at a lower dose level.

None of the newly synthesized candidates was able to achieve $100 \%$ protection in MES screen at the tested dose level in comparison with compound II. However, compound 5 offered two advantages over compound II; it induced $100 \%$ protection in scPTZ and $83 \%$ protection in MES, while compound II revealed only $50 \%$ protection in $s c$ PTZ. Moreover, compound 5 was completely safe showing $0 \%$ motor impairment in the rotarod test as compared to compound II, which produced $100 \%$ neurotoxicity.

Quantification study (estimation of $\mathrm{ED}_{50}$ ) of the most active candidates $(5,13$, and 19) in $s c$ PTZ screen has been performed accordingly. Compounds 5 (ED $45 \mathrm{mg} / \mathrm{kg}, 99 \mu \mathrm{mol} / \mathrm{kg}), 13$ (ED 40 $\mathrm{mg} / \mathrm{kg}, 109 \mu \mathrm{mol} / \mathrm{kg}$ ) and $19\left(\mathrm{ED}_{50} 81 \mathrm{mg} / \mathrm{kg}, 185 \mu \mathrm{mol} / \mathrm{kg}\right)$ with confidence limits (30.09-67.28), (35.69-64.55) and (56.68-115.75), respectively, exhibited higher potency with respect to the reference drug stiripentol (I) (ED $50115 \mathrm{mg} / \mathrm{kg}, 490 \mu \mathrm{mol} / \mathrm{kg}$ ) and lead compound III (ED $50110 \mathrm{mg} / \mathrm{kg}, 256 \mu \mathrm{mol} / \mathrm{kg})$.

Table 2: ED 50 of compounds 5, 13, and 19 in scPTZ screen

\begin{tabular}{ll}
\hline Compound & ED $_{\mathbf{5 0}} \mathbf{a}^{\mathbf{a g} / \mathbf{k g})^{\mathbf{b}}}$ \\
\hline 5 & $45(30.09-67.28)$ \\
13 & $48(35.69-64.55)$ \\
19 & $81(56.68-115.75)$ \\
Stiripentol $^{c}$ & $115(99.14-133.39)$ \\
III $^{\mathrm{c}}$ & $110(93.19-129.85)$ \\
\hline
\end{tabular}

${ }^{\mathrm{a}} \mathrm{ED}_{50}$ median effective dose required to assure anticonvulsant protection in $50 \%$ animals, bData in parentheses are the confidence limits, cadopted data from [8].

\section{Structure-activity relationships (SAR)}

The ligand-based design of candidates 3-22 mainly depends on three leads (stiripentol (I), compounds II and III). The idea was to hybridize stiripentol backbone with both semicarbazone moiety of compound II (responsible for MES activity) and the pyrazoline-Naroyl scaffold of compound III (responsible for $s c$ PTZ activity) in order to achieve wide spectrum anticonvulsant candidates.

Thus, in series A (compounds 3-10), the carbohydrazide moiety has been used as a linker between the 2-pyrazoline ring and the aroyl group of compound III. This design offered the presence of both semicarbazone-like scaffold (carbohydrazide) and pyrazoline- $\mathrm{N}$ - aroyl structure in one compound with a maximum number of free $\mathrm{NH}$ due to its impact on MES activity as proven by compound II (fig. 3). The invivo screens revealed that significant improvement against MES-induced seizures has been achieved successfully with slight enhancement against scPTZ-induced convulsions. The 4-nitro derivative, compound 5 , is the most active substituent in this series. It was 2.7 and 1.3 times more active than reference drug stiripentol and lead compound III, respectively.

This outcome directly correlates the semicarbazone-like pharmacophore (carbohydrazide) to the MES activity and postulate that the distance between 2-pyrazoline moiety and the aroyl group is not a crucial factor to achieve $100 \%$ protection in $s c$ PTZ test. 


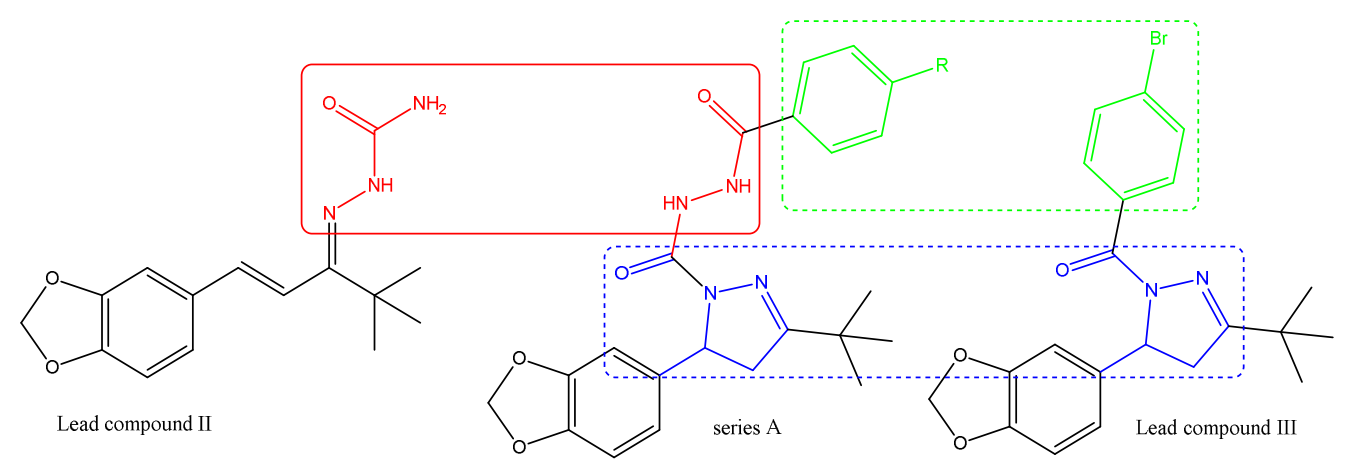

Fig. 3 Ligands based design of series $\mathrm{A}$

Semicarbazone is a well-documented pharmacophore in the design of novel anticonvulsant agents [21-23]. Thus, it was of great interest to synthesize the semicarbazone analogues of series A. Series B showed outstanding anticonvulsant activity against $s c$ PTZ at lower doses when compared to series A. Series B members, compounds $12-16$, were very effective in scPTZ screen revealing over 83 $\%$ protection. The most potent congener was the nitro derivative, compound 13, which showed $100 \%$ protection in scPTZ at 228 $\mu \mathrm{mol} / \mathrm{kg}$. It is $3.3,1.5$, and 1.2 times more active than reference drug stiripentol, lead compound III and the new compound 5, respectively. However, the MES profile of series B was not satisfactory, which could be explained by the absence of "NHCO" moiety due to the formation of semicarbazone. Noteworthy, compound $12(212 \mu \mathrm{mol} / \mathrm{kg})$ revealed a comparable protective ability as stiripentol (I, $640 \mu \mathrm{mol} / \mathrm{kg}$ ) in both MES and $s c$ PTZ screens. Therefore, it was3 times more active than compound I. These biological findings emphasized the significance of semicarbazone moiety as an anticonvulsant pharmacophore. Also, the inferior MES profile of series B clearly stressed on the relation between free $\mathrm{NH}$ and MES activity.

Studying the impact of cyclization of series A is very striking. The great change of semicarbazone-like moiety (carbohydrazide) from open-chain hydrogen donor and acceptor to electron-rich aromatic ring is expected to have a profound influence on anticonvulsant activity and will add further knowledge to the SAR of such compounds. Series C was very effective in the $s c$ PTZ screen. All members exhibited over $50 \%$ protection. Two members (19 and 21) achieved $100 \%$ protection at dose levels lower than the reference drug (I) and lead compound III, being 2.6 and 2.4 times more active than stiripentol in scPTZ, respectively. The nitro group was proposed to be the highest active substitute but in this series, the amino derivative has also displayed maximum protection in the $s c$ PTZ screen. The MES activity of series $\mathrm{C}$ was the weakest among the novel series. MES outcomes of series $\mathrm{C}$ added further clue to the importance of amidic NH in the MES activity of anticonvulsants. Slight dose differences between compounds 5 and its cyclic analogue 19 , to achieve $100 \%$ protection in the $s c$ PTZ test, highlighting the insignificance of amidic hydrogen and the impact of electron rich atoms and aryl group in such screen. The $100 \%$ protection attained by the amino-derivative (21) stressed the fact that the electron-rich aryl linker between 2-pyrazoline and aryl group was very valuable for the $s c$ PTZ screen. Moreover, the distance between 2-pyrazoline and aryl group is of less importance as proven by series A. Existence of 1,3,4-oxadiazole ring potentiated the anticonvulsant activity of compound 7 from $66 \%$ to $100 \%$ in its cyclic analogue (21).

\section{CONCLUSION}

Ligand-based design, together with molecular hybridization in drug design, succeeded to generate potent and wide spectrum candidates. Compounds 3-10, its semicarbazone analogues $12-16$, and its cyclic congeners 17-23 are proposed as potential anticonvulsant agents. Many derivatives have shown better activity than reference drug (stiripentol I) and lead compound III. Compound 5wasthe most active surrogate having highest efficacy and widest spectrum. It could act as a prospective lead for future development of broad spectrum anticonvulsants and further modification of the semicarbazone-based pharmacophoric model. Many SAR-related findings have been discovered and confirmed, such as the significance of free $\mathrm{NH}$ on the MES activity and the impact of cyclizing aroyl-carbohydrazides to 1,3,4-oxadiazoles on scPTZ screen.

\section{AUTHOR CONTRIBUTION}

M. F. El-Behairy performed the chemistry part, writing and proofreading the manuscript.

Hanan Naeim Attia conducted the pharmacological evaluation, writing and proof-reading the manuscript.

\section{CONFLICT OF INTERESTS}

Declared none

\section{REFERENCES}

1. WHO, Epilepsy Fact sheet N ${ }^{\circ} 999 ;$ Available from 2015. http://www.who.int/mediacentre/factsheets/fs999/en/. [Last accessed on 06 Feb 2017]

2. Chang BS, Lowenstein DH. Epilepsy. N Engl J Med 2003;349:1257-66.

3. Kamiński K, Rapacz A, Łuszczki JJ, Latacz G, Obniska J, KiećKononowicz K, et al. Design, synthesis and biological evaluation of new hybrid anticonvulsants derived from N-benzyl-2-(2,5dioxopyrrolidin-1-yl)propanamide and 2-(2,5-dioxopyrrolidin-1yl)butanamide derivatives. Bioorg Med Chem 2015;23:2548-61.

4. Perucca E, French J, Bialer M. Development of new antiepileptic drugs: challenges, incentives, and recent advances. Lancet Neurol 2007;6:793-804.

5. Cramer JA, Mintzer S, Wheless J, Mattson RH. Adverse effects of antiepileptic drugs: a brief overview of important issues. Expert Rev Neurother 2010;10:885-91.

6. Khan HN, Kulsoom S, Rashid H. Ligand-based pharmacophore model development for the identification of a novel antiepileptic compound. Epilepsy Res 2012;98:62-71.

7. Stiripentol. BCX 2600. Drugs RD 2002;3:220-2.

8. Aboul-Enein MN, El-Azzouny AA, Attia MI, Maklad YA, Amin $\mathrm{KM}$, Abdel-Rehim $\mathrm{M}$, et al. Design and synthesis of novel stiripentol analogues as potential anticonvulsants. Eur J Med Chem 2012;47:360-9.

9. Rajak H, Deshmukh R, Veerasamy R, Sharma AK, Mishra P, Kharya MD. Novel semicarbazones based 2,5-disubstituted-1,3,4oxadiazoles: one more step towards establishing four binding sites pharmacophoric model hypothesis for anticonvulsant activity. Bioorg Med Chem Lett 2010;20:4168-72.

10. Harish KP, Mohana KN, Mallesha L, Prasanna k, Basavapatna N. Synthesis of novel 1-[5-(4-methoxy-phenyl)-[1,3,4]oxadiazol-2yl]-piperazine derivatives and evaluation of their in vivo anticonvulsant activity. Eur J Med Chem 2013;65:276-83.

11. Rajak H, Singh T, Bhupendra S, Avineesh R, Kamlesh S, Anil K, et al. Novel limonene and citral-based 2,5-disubstituted-1,3,4oxadiazoles: a natural product coupled approach to semicarbazones for antiepileptic activity. Bioorg Med Chem Lett 2013;23:8648. 
12. Pandeya SN, Ponnilavarasan I, Pandey A, Lakhan R, Stables JP. Evaluation of p-nitrophenyl substituted semicarbazones for anticonvulsant properties. Pharmazie 1999;54:923-5.

13. Dimmock JR, Vashishtha SC, Stables JP. Anticonvulsant properties of various phenylhydrazones, oxamoylhydrazones and semicarbazones derived from aromatic and unsaturated carbonyl compounds. Eur J Med Chem 2000;35:241-8.

14. Krall RL, Penry JK, White BG, Kupferberg HJ, Swinyard EA. Antiepileptic drug development: II. Anticonvulsant drug screening. Epilepsia 1978;19:409-28.

15. Alam O, Mullick P, Verma SP, Gilani SJ, Khan SA, Siddiqui N, et al. Synthesis, anticonvulsant and toxicity screening of newer pyrimidine semicarbazone derivatives. Eur J Med Chem 2010;45:2467-72.

16. Luszczki JJ, Czuczwar M, Gawlik P, Sawiniec-Pozniak G, Czuczwar K, Czuczwar SJ. 7-Nitroindazole potentiates the anticonvulsant action of some second-generation antiepileptic drugs in the mouse maximal electroshock-induced seizure model. J Neural Transm 2006;113:1157-68.

17. Dunham NW, Miya TS. A note on a simple apparatus for detecting neurological deficit in rats and mice. J Am Pharm Assoc 2006;46:208-9.

18. Augustine JK, Vairaperumal V, Narasimhan S, Alagarsamy P, Radhakrishnan A. Propylphosphonic anhydride (T3P®): an efficient reagent for the one-pot synthesis of 1,2,4-oxadiazoles, 1,3,4-oxadiazoles, and 1,3,4-thiadiazoles. Tetrahedron 2009; 65:9989-96.

19. Löscher W. Critical review of current animal models of seizures and epilepsy used in the discovery and development of new antiepileptic drugs. Seizure 2011;20:359-68.

20. White HS. Preclinical development of antiepileptic drugs: past, present, and future directions. Epilepsia 2003;44:2-8.

21. Pandeya SN, Raja AS, Stables JP. Synthesis of isatin semicarbazones as novel anticonvulsants- the role of hydrogen bonding. J Pharm Pharm Sci 2002;5:266-71.

22. Yogeeswari $P$, Ragavendran JV, Thirumurugan $R$, Induja $S$, Sriram D, Stables JP. Synthesis and structure-activity relationship on anticonvulsant aryl semicarbazones. Med Chem 2006;2:55-62.

23. Dimmock JR, Puthucode RN, Smith JM, Hetherington M, Quail JW, Pugazhenthi U, et al. (Aryloxy)aryl semicarbazones and related compounds: a novel class of anticonvulsant agents possessing high activity in the maximal electroshock screen. J Med Chem 1996;39:3984-97.

\section{How to cite this article}

- Mohammed Farrag El-Behairy, Hanan Naeim Hafez Attia. Design, synthesis and anticonvulsant profile of 5(benzo[D][1,3]dioxol-5-YL)-3-tert-butyl-4,5-dihydropyrazole derivatives. Int J Pharm Pharm Sci 2017;9(6):180-188. 